\title{
Putrescine-functionalized carbon quantum dot (put-CQD) nanoparticles effectively prime grapevine (Vitis vinifera cv. 'Sultana') against salt stress
}

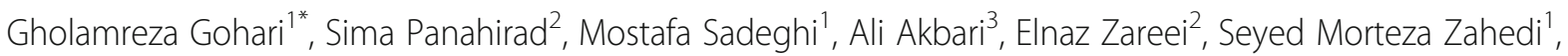
Mohammad Kazem Bahrami ${ }^{4}$ and Vasileios Fotopoulos ${ }^{5}$

\begin{abstract}
Background: Salinity is an important global problem with destructive impacts on plants leading to different biochemical and metabolic changes in plants through induced oxidative stress that disturbs metabolism, growth, performance and productivity of plants. Given that putrescine (Put) and carbon quantum dots (CQDs), individually, have promising effects in different plant processes, the idea of their combination in a nano-structure "Put-CQD" lead to its synthesis to evaluate the potential exertion of synergistic effects. The current study aimed to investigate the application of newly-synthesized nanoparticles (NPs) consisting of CQDs and Put in grapevine (Vitis vinifera cV. 'Sultana') under salinity stress conditions. For this purpose, Put, CQDs and Put-CQD NPs at 5 and $10 \mathrm{mg} \mathrm{L}^{-1}$ concentrations were applied as chemical priming agents in 'Sultana' grapevine $48 \mathrm{~h}$ prior salinity stress imposition (0 and $100 \mathrm{mM} \mathrm{NaCl}$ ).
\end{abstract}

Results: Salinity significantly decreased $(P \leq 0.05)$ morphological parameters, photosynthetic pigments, chlorophyll fluorescence parameters and membrane stability index. In addition, salinity enhanced $\mathrm{MDA}, \mathrm{H}_{2} \mathrm{O}_{2}$, proline content and antioxidant enzyme activity. Results revealed that Put-CQD NPs, particularly at $10 \mathrm{mg} \mathrm{L}^{-1}$ concentration, alleviated the destructive impacts of salinity stress by improving leaf fresh and dry weights, $\mathrm{K}^{+}$content, photosynthetic pigments, chlorophyll fluorescence and SPAD parameters, proline content, total phenolics and antioxidant enzymatic activities (CAT, APX, GP and SOD), while decreasing $\mathrm{Na}^{+}$content, EL, MDA and $\mathrm{H}_{2} \mathrm{O}_{2}$ levels.

Conclusion: To conclude, Put-CQD NPs represent an innovative priming treatment that could be effectively applied on grapevine to improve plant performance under salinity stress conditions.

Keywords: Carbon quantum dots, Grapevine, Nanotechnology, Priming, Abiotic stress

\footnotetext{
* Correspondence: gohari.gh@maragheh.ac.ir

'Department of Horticultural Sciences, Faculty of Agriculture, University of Maragheh, Maragheh, Iran

Full list of author information is available at the end of the article
}

(c) The Author(s). 2021 Open Access This article is licensed under a Creative Commons Attribution 4.0 International License, which permits use, sharing, adaptation, distribution and reproduction in any medium or format, as long as you give appropriate credit to the original author(s) and the source, provide a link to the Creative Commons licence, and indicate if changes were made. The images or other third party material in this article are included in the article's Creative Commons licence, unless indicated otherwise in a credit line to the material. If material is not included in the article's Creative Commons licence and your intended use is not permitted by statutory regulation or exceeds the permitted use, you will need to obtain permission directly from the copyright holder. To view a copy of this licence, visit http://creativecommons.org/licenses/by/4.0/ The Creative Commons Public Domain Dedication waiver (http://creativecommons.org/publicdomain/zero/1.0/) applies to the data made available in this article, unless otherwise stated in a credit line to the data. 


\section{Background}

Abiotic stress factors such as salinity and drought are affecting most agricultural lands, limiting plant distribution in habitats. Salinity stress, a major environmental restrain and a key global climate change-related problem, causes negative impacts on performance and yield of plants [1, 2]. Substantial disorders in morphological, physiological, nutritional and biochemical characteristics, ion toxicity or imbalance $\left(\mathrm{Na}^{+}\right.$and $\left.\mathrm{Cl}^{-}\right)$and osmotic stress are some of the main salinity effects in plants [3]. As a result, salinity stress leads to numerous biochemical and metabolic changes resulting in oxidative stress in plants [2, 4].

Chemical priming is amongst several approaches employed to deal with stresses like salinity, as an advantageous technique to increase plant tolerance to different stresses [5, 6], before their occurrence [7]. Natural compounds such as amino acids (e.g. proline and glycinebetaine [8];) and hormones (e.g. salicylic acid [9];), as well as synthetic molecules (e.g. NOSH-aspirin [10];), are amongst a number of materials with the potential of acting as priming agents against stresses. Moreover, certain chemicals (e.g., [11]) and nanoparticles (NPs) (e.g., $[12,13])$ could ameliorate the negative effects of salinity stress through priming.

Abundant benefits such as superior performance of chemicals via improved nano-structure are proposed by nanotechnology, which then cause reduction in their environmental load via chemical priming application [14]. Therefore, their application represents a promising strategy for agricultural industries [15]. NPs have great impact on plant growth and development, as well as on plant tolerance to abiotic stresses through their effectiveness in ROS detoxification [16]. Furthermore, photosynthesis, as a cellular process sensitive to abiotic stresses, could be protected by NP application by diminishing osmotic and oxidative stresses [14]. Subsequently, NP application has been attracting increasing attention in this regard. Quantum dot (QD) NPs are $2-10 \mathrm{~nm}$ in size, giving them the role of a carrier and size-dependent essential properties. They are zero dimensional and semiconductor NPs with quantization of energy [17]. In addition, QDs are extensively applied in biological studies for subcellular labeling and imaging through their distinctive fluorescent characteristics [18]. Carbon QDs (CQDs), as a new generation of QD NPs in which carbonic compounds contain oxygen, show somewhat different properties than carbon-based NPs [19]. CQD NPs are less than $10 \mathrm{~nm}$ in size (like other QDs) with round shape giving them unique physicochemical properties (e.g., low or no toxicity and high water solubility, biocompatibility and biodegradability) with enormous range of usage [20]. Some studies have demonstrated advantageous impacts of different QDs in plants; graphene QDs caused an increase in growth parameters (e.g., leaves, roots, shoots, flowers and fruits) of treated garlic and coriander seeds [21]. CQD NPs enhanced rice yield due to increased RuBisCO enzyme activity. In addition, they improved seed germination, root elongation, carbohydrate production and resistance to diseases all through enhanced thionin gene expression [22].

Putrescine (Put), as one of major polyamines (PAs), has crucial functions in plant growth and differentiation and also response to stresses with salt stress in particular [1, 23-25]. Put stabilizes biological membranes and macromolecular structures of cells [26]. With low molecular weight and polycationic nature [27, 28], Put plays important roles in numerous physiological and developmental processes such as cell division, rhizogenesis, embryogenesis, senescence, floral development and fruit setting and ripening. It is worth stating that Put concentration increases under stress conditions to enhance plant tolerance to the stressor [26]. Such an increasing trend in Put content was reported in plants under salt stress [29]. Put application enhances stress tolerance by stabilizing membrane and cellular structures, scavenging free radicals, modulating ion channels, maintaining the cation-anion balance and energizing cells via stimulating of ATP synthesis [24, 28]. Therefore, exogenous Put treatment could be considered as a typical attempt to improve plant performance under salinity, mostly by enhancing photosynthetic efficiency and preventing chlorophyll loss [1]. Plant species, duration and intensity of stress, developmental stage of plant tissues and applied treatments could affect Put content of plants under stress conditions. Response to the stress condition by Put demonstrates its role as a signaling molecule [26]. Put could reverse growth inhibition caused by stress, decrease cell membrane damage, lipid peroxidation, ROS accumulation, increase in $\mathrm{Na}^{+}$and $\mathrm{Cl}^{-}$and loss of chlorophyll, and also increase expression of osmotically responsive genes, antioxidant enzymatic activities, nonenzymatic compounds and compatible osmolytes [29]. Shu et al. [1] noticed that Put treatment increased Put content in plants under salinity conditions. Put application decreases stress and lipid peroxidation damage of plant under salt stress by improving plant growth and antioxidant enzyme activity, inhibiting $\mathrm{Na}^{+}$and $\mathrm{Cl}^{-}$uptake and accelerating accumulation of $\mathrm{K}^{+}, \mathrm{Ca}^{2+}$ and $\mathrm{Mg}^{2+}[30]$.

Grapevine (Vitis vinifera L.) is considered as one of the most important, added-value agricultural products cultivated worldwide, consequently with significant economic importance [31]. Grapevine, relatively sensitive to salt stress, suffers significant losses in growth and productivity and also fruit quality under salinity conditions [32].

Considering the protective properties of CQD NPs and Put when individually applied, especially at lessening 
destructive effects of salinity stress, conjugating Put with CQD NPs (Put-CQD NPs) could potentially improve entrance of Put inside plant cells and thus improve its efficiency, particularly at lower doses. Consequently, after synthesizing Put-CQD NPs, NPs were applied as priming agents in grapevine (Vitis vinifera cv. 'Sultana') to alleviate the undesirable impacts of salt stress conditions, representing the first report of its kind to our knowledge.

\section{Results}

Put-CQDs synthesis and characterization

One-pot and easy hydrothermal method was used in the preparation of Put-CQD NPs. Citric acid is the most commonly used carbon source which could be utilized alone or with other functionalized amines for CQDs preparation. As can be seen from Fig. 1, the carboxylic acid groups of citric acid firstly condensed with the amine groups of putrescines leading to form polymerlike CQDs, which were then carbonized to form the CDs. The structure composition and morphology of Synthesized Put-CQD NPs were characterized using FTIR and TEM analysis. In the FTIR spectrum of PutCQD NPs (Fig. 2a), characteristic bands at $3480 \mathrm{~cm}^{-1}$ and $2980 \mathrm{~cm}^{-1}$ corresponded to the stretching vibrations of $\mathrm{O}-\mathrm{H}$ and $\mathrm{C}-\mathrm{H}$, respectively. The sharp bands at $1557 \mathrm{~cm}^{-1}$ and $1389 \mathrm{~cm}^{-1}$ could be related to bending<smiles>CC(C)(C)C(=O)OCC(O)(CC(=O)[O-])C(=O)OC(=O)CC(O)(CC(=O)O)C(=O)O</smiles>

Citric Acid

Putrescine
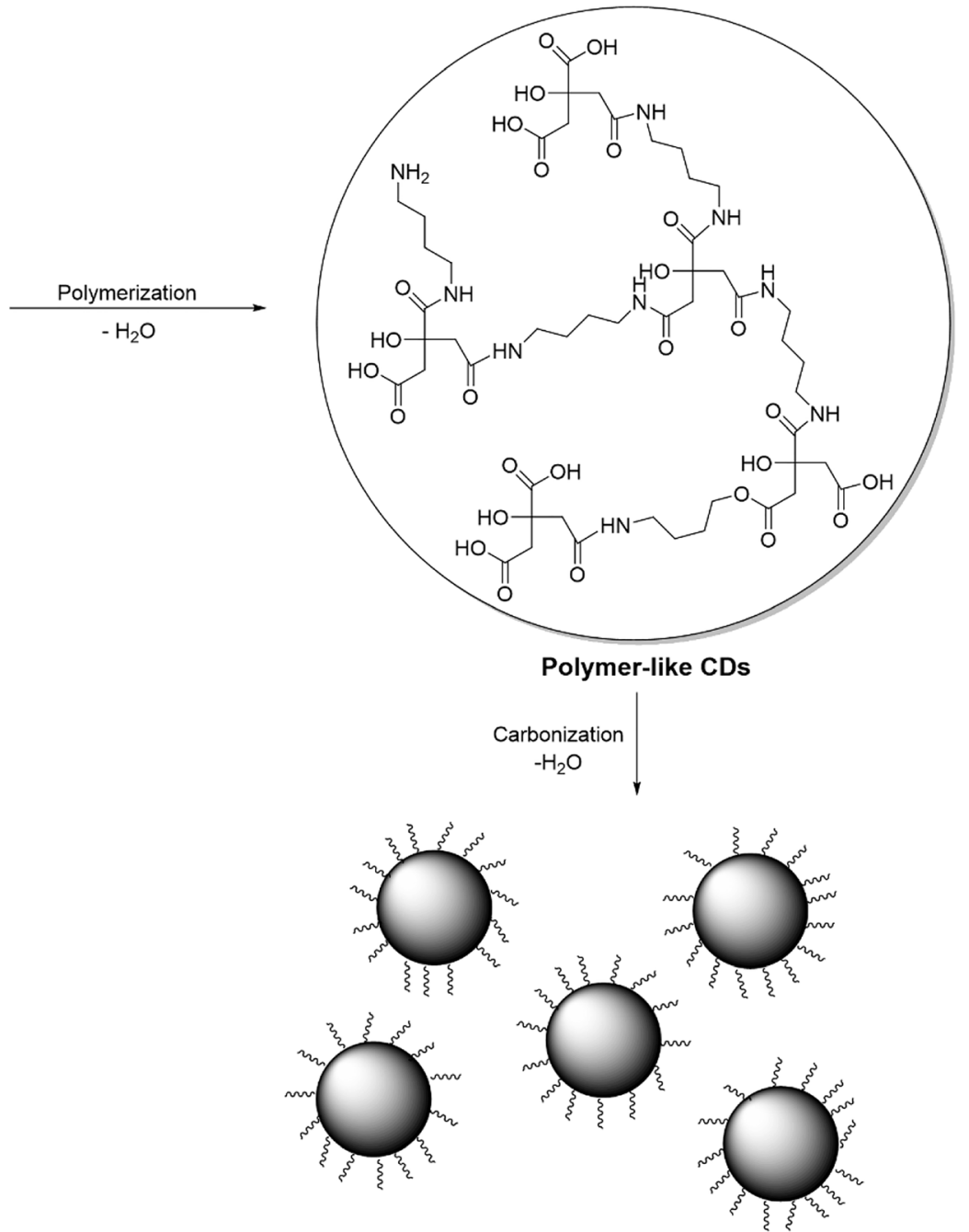

Fig. 1 A synthetic step of Put-CQD NPs: from ionization to condensation, polymerization, and carbonization 
A

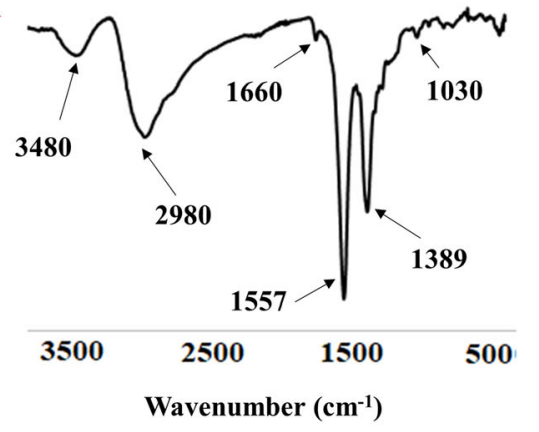

B

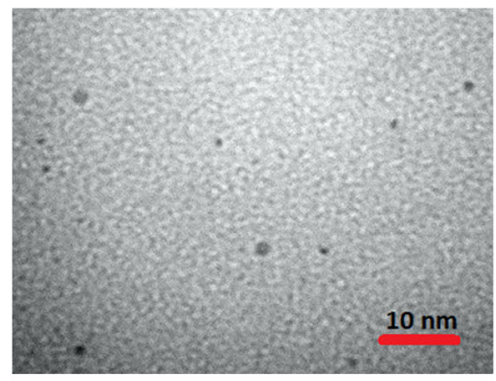

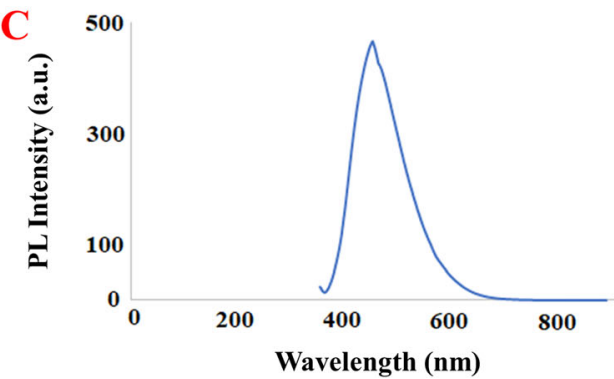

Fig. 2 FTIR spectrum (a), TEM image (b) and PL spectrum (c) of synthesized Put-CQD NPs

vibration of $\mathrm{N}-\mathrm{H}$ and $\mathrm{C}-\mathrm{NH}-\mathrm{C}$, respectively. Moreover, $\mathrm{C}=\mathrm{O}$ and $\mathrm{C}-\mathrm{N}$ stretching vibration could be seen at $1660 \mathrm{~cm}^{-1}$ and $1030 \mathrm{~cm}^{-1}$. Figure 2b illustrates TEM image of Put-CQD NPs. As it could be seen, the synthesized Put-CQD NPs have uniform dispersion without significant aggregation and particle size in the range of 3-5 $\mathrm{nm}$. Furthermore, the PL spectrum shows blue fluorescence with $\lambda_{\max }$ at $440 \mathrm{~nm}$ under $360 \mathrm{~nm}$ UVlight (Fig. 2c).

\section{Leaf FW and DW}

Salinity negatively affected leaf FW and DW. Leaf FW increased significantly $(P \leq 0.05)$ by $10 \mathrm{mg} \mathrm{L}^{-1}$ Put and 5 and $10 \mathrm{mg} \mathrm{L}^{-1}$ Put-CQD NPs; however, the other treatments had no effect or decreased the weight under control conditions. All treatments enhanced leaf DW under control conditions. Under salt stress condition, $10 \mathrm{mg}$ $\mathrm{L}^{-1}$ CQD NPs and 5 and $10 \mathrm{mg} \mathrm{L}^{-1}$ Put-CQD NPs increased leaf FW, while the other priming treatments exerted no effect compared with unprimed grapevines under salinity. All priming treatments enhanced leaf DW under salinity condition. Under both conditions, 10 $\mathrm{mg} \mathrm{L}^{-1}$ Put-CQD NPs could be considered as the optimal priming treatment (Table 1).

\section{Ionic homeostasis}

Salinity significantly decreased $(P \leq 0.05) \mathrm{K}^{+}$content and increased $\mathrm{Na}^{+}$content and $\mathrm{Na}^{+} / \mathrm{K}^{+}$ratio. Under non saline conditions, all treatments lowered $\mathrm{Na}^{+}$content compared with the control and $\mathrm{K}^{+}$content was only enhanced in grapevine primed by $10 \mathrm{mg} \mathrm{L}^{-1}$ Put-CQD NPs. Consequently, all priming treatments increased the $\mathrm{Na}^{+} / \mathrm{K}^{+}$ratio, with the exception of $5 \mathrm{mg} \mathrm{L}^{-1}$ put and 10 $\mathrm{mg} \mathrm{L}^{-1}$ CQDs which had no effect compared with the control. Under salinity conditions, all treatments decreased $\mathrm{Na}^{+}$content and $\mathrm{Na}^{+} / \mathrm{K}^{+}$ratios, while Put and Put-CQD NPs treatments (5 and $10 \mathrm{mg} \mathrm{L}^{-1}$ ) increased $\mathrm{K}^{+}$content. Put-CQD NPs at $10 \mathrm{mg} \mathrm{L}^{-1}$ concentration demonstrated the best results for $\mathrm{K}^{+}, \mathrm{Na}^{+}$and $\mathrm{Na}^{+} / \mathrm{K}^{+}$ ratio under both conditions (Table 1).

\section{Physiological parameters}

Chl $a, b$ and carotenoid content were decreased by salinity. Significant increase $(P \leq 0.05)$ in Chl $a$ and $b$ content was observed following Put and Put-CQD NPs priming treatments; all priming treatments enhanced carotenoid content under control conditions compared with the control. Under stress conditions, all treatments significantly increased Chl $a, b$ and carotenoid content. Put-CQD NPs at $10 \mathrm{mg} \mathrm{L}^{-1}$ lead to the highest value for Chl $a, b$ and carotenoid levels under both control and stress conditions. SPAD value was negatively affected by salinity. With the exception of CQDs at $10 \mathrm{mg} \mathrm{L}^{-1}$ which showed no significant difference to the control, other treatments enhanced SPAD values under non-stress conditions. Increase in SPAD values was additionally achieved by Put ( 5 and 10 $\mathrm{mg} \mathrm{L}^{-1}$ ), CQDs (10 $\mathrm{mg} \mathrm{L}^{-1}$ ) and Put-CQD NPs (5 and 10 $\mathrm{mg} \mathrm{L}^{-1}$ ) priming treatments under salt stress conditions. 
Table 1 Effect of different concentrations of Put, CQDs, and Put-CQD NPs on leaf fresh (FW) and dry (DW) weights, $\mathrm{Na}^{+}$and $\mathrm{K}^{+}$ concentrations and ratio of Vitis vinifera cv. 'Sultana' under salinity stress (CQDs, carbon quantum dots; Put, putrescine, Put-CQD NPs, carbon quantum dots functionalized by putrescine nanoparticles). Different letters indicate significant differences based on Duncan's post-hoc analysis at $P \leq 0.05$

\begin{tabular}{|c|c|c|c|c|c|}
\hline Treatments & $\begin{array}{l}\text { Leaf FW } \\
\text { (g) }\end{array}$ & $\begin{array}{l}\text { Leaf DW } \\
\text { (g) }\end{array}$ & $\begin{array}{l}\mathrm{Na}^{+} \\
\left(\mathrm{mM} \mathrm{kg}^{-1}\right)\end{array}$ & $\begin{array}{l}\mathrm{K}^{+} \\
\left(\mathrm{mM} \mathrm{kg}^{-1}\right)\end{array}$ & $\mathrm{Na}^{+} / \mathrm{K}^{+}$ \\
\hline $\mathrm{NaCl}(0 \mathrm{mM})+\mathrm{No}$ Treatment & $83.61 \pm 0.21^{\text {de }}$ & $7.15 \pm 0.03^{e}$ & $0.212 \pm 0.019^{\mathrm{ef}}$ & $5.64 \pm 0.91^{b c}$ & $0.037 \pm 0.003^{f}$ \\
\hline $\mathrm{NaCl}(0 \mathrm{mM})+$ Put $5 \mathrm{mg} \mathrm{L}^{-1}$ & $85.23 \pm 0.08^{d}$ & $8.51 \pm 0.01^{d}$ & $0.185 \pm 0.014^{9}$ & $5.72 \pm 0.61^{b c}$ & $0.032 \pm 0.009^{f g}$ \\
\hline $\mathrm{NaCl}(0 \mathrm{mM})+$ Put $10 \mathrm{mg} \mathrm{L}^{-1}$ & $87.54 \pm 0.15^{c}$ & $8.92 \pm 0.08^{\mathrm{cd}}$ & $0.173 \pm 0.013^{g h}$ & $5.87 \pm 0.83^{b}$ & $0.029 \pm 0.005^{9}$ \\
\hline $\mathrm{NaCl}(0 \mathrm{mM})+\mathrm{CQDs} 5 \mathrm{mg} \mathrm{L}^{-1}$ & $81.39 \pm 0.41^{f}$ & $9.55 \pm 0.07^{c}$ & $0.202 \pm 0.017^{f}$ & $4.96 \pm 0.43^{c}$ & $0.048 \pm 0.007^{\mathrm{e}}$ \\
\hline $\mathrm{NaCl}(0 \mathrm{mM})+\mathrm{CQDs} 10 \mathrm{mg} \mathrm{L}^{-1}$ & $85.08 \pm 0.38 d^{d}$ & $10.06 \pm 0.09^{b c}$ & $0.191 \pm 0.011^{\mathrm{fg}}$ & $5.51 \pm 0.67^{\mathrm{bc}}$ & $0.034 \pm 0.004^{f g}$ \\
\hline $\mathrm{NaCl}(0 \mathrm{mM})+$ Put-CQD NPs $5 \mathrm{mg} \mathrm{L}^{-1}$ & $89.87 \pm 0.29^{b}$ & $10.15 \pm 0.05^{b}$ & $0.171 \pm 0.008^{g h}$ & $6.16 \pm 0.89^{a b}$ & $0.027 \pm 0.009^{9}$ \\
\hline $\mathrm{NaCl}(0 \mathrm{mM})+$ Put-CQD NPs $10 \mathrm{mg} \mathrm{L}^{-1}$ & $92.09 \pm 0.61^{\mathrm{a}}$ & $11.01 \pm 0.03^{\mathrm{a}}$ & $0.163 \pm 0.016^{h}$ & $7.08 \pm 0.69^{a}$ & $0.023 \pm 0.004^{h}$ \\
\hline $\mathrm{NaCl}(100 \mathrm{mM})+$ No Treatment & $65.36 \pm 0.2^{i j}$ & $4.01 \pm 0.04^{i}$ & $0.461 \pm 0.016^{\mathrm{a}}$ & $2.58 \pm 0.28^{f}$ & $0.178 \pm 0.006^{\mathrm{a}}$ \\
\hline $\mathrm{NaCl}(100 \mathrm{mM})+$ Put $5 \mathrm{mg} \mathrm{L}^{-1}$ & $65.05 \pm 0.19^{j}$ & $4.85 \pm 0.03^{h}$ & $0.355 \pm 0.019^{b}$ & $3.12 \pm 0.81^{\mathrm{e}}$ & $0.113 \pm 0.008^{b}$ \\
\hline $\mathrm{NaCl}(100 \mathrm{mM})+$ Put $10 \mathrm{mg} \mathrm{L}^{-1}$ & $68.23 \pm 0.08^{i j}$ & $5.17 \pm 0.09^{9}$ & $0.258 \pm 0.006^{\mathrm{de}}$ & $3.89 \pm 0.97^{\text {de }}$ & $0.066 \pm 0.005^{d}$ \\
\hline $\mathrm{NaCl}(100 \mathrm{mM})+\mathrm{CQDs} 5 \mathrm{mg} \mathrm{L}^{-1}$ & $71.02 \pm 0.19^{i}$ & $5.82 \pm 0.02^{\mathrm{fg}}$ & $0.321 \pm 0.017^{b c}$ & $2.74 \pm 0.61^{\mathrm{ef}}$ & $0.117 \pm 0.003^{b}$ \\
\hline $\mathrm{NaCl}(100 \mathrm{mM})+\mathrm{CQDs} 10 \mathrm{mg} \mathrm{L}^{-1}$ & $74.91 \pm 0.27^{h}$ & $6.07 \pm 0.06^{f}$ & $0.309 \pm 0.014^{c}$ & $2.98 \pm 0.81^{\mathrm{ef}}$ & $0.103 \pm 0.007^{c}$ \\
\hline $\mathrm{NaCl}(100 \mathrm{mM})+$ Put-CQD NPs $5 \mathrm{mg} \mathrm{L}^{-1}$ & $79.15 \pm 0.43^{9}$ & $7.88 \pm 0.06^{\mathrm{de}}$ & $0.284 \pm 0.007^{d}$ & $4.13 \pm 0.65^{d}$ & $0.068 \pm 0.008^{d}$ \\
\hline $\mathrm{NaCl}(100 \mathrm{mM})+$ Put-CQD NPs $10 \mathrm{mg} \mathrm{L}^{-1}$ & $82.34 \pm 0.31^{e}$ & $7.97 \pm 0.04^{\text {de }}$ & $0.229 \pm 0.009^{e}$ & $3.93 \pm 0.75^{\text {de }}$ & $0.058 \pm 0.004^{\mathrm{de}}$ \\
\hline
\end{tabular}

The highest SPAD value was recorded at $10 \mathrm{mg} \mathrm{L}^{-1}$ PutCQD NPs-primed grapevines (Table 2).

Chlorophyll fluorescence parameters were significantly negatively affected after imposing salinity. ${ }^{\mathrm{Fv}} / \mathrm{Fm}$ was increased by all treatments, with the sole exception of 5 $\mathrm{mg} \mathrm{L}^{-1}$ CQDs priming treatment which showed no difference to unprimed, unstressed samples. All priming treatments positively affected $\mathrm{Fv} / \mathrm{Fm}$ under stress conditions. Put and Put-CQD NPs priming treatments increased ${ }^{\mathrm{Fv}} / \mathrm{Fo}$ parameter under control and stress conditions. Regarding Y (II) parameter, Put-CQD NP treatments (5 and $10 \mathrm{mg} \mathrm{L}^{-1}$ ) leads to its significant increase $(P \leq 0.05)$ under control conditions. Under salinity stress, all treatments enhanced Y (II) parameter. Considering all parameters, Put-CQD NPs at $10 \mathrm{mg} \mathrm{L}^{-1}$ concentration represented the optimal treatment

Table 2 Effect of different concentrations of Put, CQDs and Put-CQD NPs on photosynthetic pigments and SPAD index of Vitis vinifera cv. 'Sultana' under salinity stress (CQDs, carbon quantum dots; Put, putrescine, Put-CQD NPs, carbon quantum dots functionalized by putrescine nanoparticles). Different letters indicate significant differences based on Duncan's post-hoc analysis at $P \leq 0.05$

\begin{tabular}{|c|c|c|c|c|}
\hline Treatments & $\begin{array}{l}\mathrm{Chl} a \\
\left(\mathrm{mg} \mathrm{g}^{-1} \mathrm{FW}\right)\end{array}$ & $\begin{array}{l}\mathrm{Chl} \mathrm{b} \\
\left(\mathrm{mg} \mathrm{g}^{-1} \mathrm{FW}\right)\end{array}$ & Carotenoids $\left(\mathrm{mg} \mathrm{g}^{-1} \mathrm{FW}\right)$ & SPAD \\
\hline $\mathrm{NaCl}(0 \mathrm{mM})+$ No Treatment & $2.039 \pm 0.05^{\mathrm{ef}}$ & $0.579 \pm 0.002^{\text {cd }}$ & $0.521 \pm 0.003^{e}$ & $26.86 \pm 0.73^{c}$ \\
\hline $\mathrm{NaCl}(0 \mathrm{mM})+$ Put $5 \mathrm{mg} \mathrm{L}^{-1}$ & $2.228 \pm 0.02^{c}$ & $0.657 \pm 0.008^{b}$ & $0.633 \pm 0.012^{\mathrm{bc}}$ & $29.38 \pm 0.83^{\mathrm{ab}}$ \\
\hline $\mathrm{NaCl}(0 \mathrm{mM})+$ Put $10 \mathrm{mg} \mathrm{L}^{-1}$ & $2.317 \pm 0.12^{\mathrm{bc}}$ & $0.682 \pm 0.011^{\mathrm{ab}}$ & $0.662 \pm 0.009^{b}$ & $29.91 \pm 0.54^{\mathrm{ab}}$ \\
\hline $\mathrm{NaCl}(0 \mathrm{mM})+\mathrm{CQDs} 5 \mathrm{mg} \mathrm{L}^{-1}$ & $1.797 \pm 0.09^{f g}$ & $0.538 \pm 0.009^{d}$ & $0.584 \pm 0.011^{c d}$ & $28.13 \pm 0.91^{b}$ \\
\hline $\mathrm{NaCl}(0 \mathrm{mM})+\mathrm{CQDs} 10 \mathrm{mg} \mathrm{L}^{-1}$ & $1.983 \pm 0.01^{\mathrm{ef}}$ & $0.582 \pm 0.023^{\mathrm{cd}}$ & $0.604 \pm 0.013^{c}$ & $25.83 \pm 0.95^{\mathrm{cc}}$ \\
\hline $\mathrm{NaCl}(0 \mathrm{mM})+$ Put-CQD NPs $5 \mathrm{mg} \mathrm{L}^{-1}$ & $2.324 \pm 0.08^{b}$ & $0.697 \pm 0.016^{b}$ & $0.649 \pm 0.008^{\mathrm{bc}}$ & $29.26 \pm 0.38^{\mathrm{ab}}$ \\
\hline $\mathrm{NaCl}(0 \mathrm{mM})+$ Put-CQD NPs $10 \mathrm{mg} \mathrm{L}^{-1}$ & $2.426 \pm 0.05^{\mathrm{a}}$ & $0.723 \pm 0.008^{a}$ & $0.721 \pm 0.009^{a}$ & $31.82 \pm 0.77^{\mathrm{a}}$ \\
\hline $\mathrm{NaCl}(100 \mathrm{mM})+$ No Treatment & $1.482 \pm 0.12^{h}$ & $0.357 \pm 0.014^{f}$ & $0.358 \pm 0.007^{f}$ & $19.63 \pm 0.14^{9}$ \\
\hline $\mathrm{NaCl}(100 \mathrm{mM})+$ Put $5 \mathrm{mg} \mathrm{L}^{-1}$ & $1.718 \pm 0.07^{\mathrm{fg}}$ & $0.477 \pm 0.021^{e}$ & $0.597 \pm 0.012^{c d}$ & $21.7 \pm 0.54^{\mathrm{ef}}$ \\
\hline $\mathrm{NaCl}(100 \mathrm{mM})+$ Put $10 \mathrm{mg} \mathrm{L}^{-1}$ & $1.891 \pm 0.11^{f}$ & $0.534 \pm 0.016^{d}$ & $0.612 \pm 0.018^{c}$ & $22.7 \pm 1.12^{\mathrm{e}}$ \\
\hline $\mathrm{NaCl}(100 \mathrm{mM})+\mathrm{CQDs} 5 \mathrm{mg} \mathrm{L}^{-1}$ & $1.686 \pm 0.09^{9}$ & $0.456 \pm 0.009^{e}$ & $0.523 \pm 0.014^{\mathrm{de}}$ & $20.13 \pm 0.82^{f g}$ \\
\hline $\mathrm{NaCl}(100 \mathrm{mM})+\mathrm{CQDs} 10 \mathrm{mg} \mathrm{L}^{-1}$ & $1.751 \pm 0.08^{\mathrm{fg}}$ & $0.461 \pm 0.015^{\mathrm{e}}$ & $0.542 \pm 0.009^{d}$ & $21.4 \pm 0.61^{f}$ \\
\hline $\mathrm{NaCl}(100 \mathrm{mM})+$ Put-CQD NPs $5 \mathrm{mg} \mathrm{L}^{-1}$ & $2.052 \pm 0.01^{e}$ & $0.596 \pm 0.022^{c}$ & $0.648 \pm 0.019^{b c}$ & $22.23 \pm 1.24^{\mathrm{ef}}$ \\
\hline $\mathrm{NaCl}(100 \mathrm{mM})+$ Put-CQD NPs $10 \mathrm{mg} \mathrm{L}^{-1}$ & $2.141 \pm 0.08^{d}$ & $0.586 \pm 0.008^{c d}$ & $0.663 \pm 0.008^{b}$ & $24.2 \pm 0.43^{d}$ \\
\hline
\end{tabular}


Table 3 Effect of different concentrations of Put, CQDs, and Put-CQD NPs on chlorophyll fluorescence parameters of Vitis vinifera cV. 'Sultana' under salinity stress (CQDs, carbon quantum dots; Put, putrescine, Put-CQD NPs, carbon quantum dots functionalized by putrescine nanoparticles). Different letters indicate significant differences based on Duncan's post-hoc analysis at $P \leq 0.05$

\begin{tabular}{|c|c|c|c|c|c|c|}
\hline Treatments & Fv & Fm & Fo & $\mathrm{Fv} / \mathrm{Fm}$ & $\mathrm{Fv} / \mathrm{Fo}$ & $\mathrm{Y}(\mathrm{II})$ \\
\hline $\mathrm{NaCl}(0 \mathrm{mM})+$ No Treatment & $2.455 \pm 0.09^{c}$ & $2.631 \pm 0.08^{d}$ & $1.072 \pm 0.08^{\mathrm{ab}}$ & $0.933 \pm 0.03^{d}$ & $2.291 \pm 0.01^{d}$ & $0.674 \pm 0.01^{c}$ \\
\hline $\mathrm{NaCl}(0 \mathrm{mM})+$ Put $5 \mathrm{mg} \mathrm{L}^{-1}$ & $2.567 \pm 0.03^{\mathrm{ab}}$ & $2.252 \pm 0.07^{e}$ & $1.069 \pm 0.04^{\mathrm{ab}}$ & $1.139 \pm 0.01^{c}$ & $2.401 \pm 0.04^{c}$ & $0.672 \pm 0.02^{c}$ \\
\hline $\mathrm{NaCl}(0 \mathrm{mM})+$ Put $10 \mathrm{mg} \mathrm{L}^{-1}$ & $2.579 \pm 0.06^{\mathrm{ab}}$ & $2.137 \pm 0.09^{9}$ & $1.021 \pm 0.02^{\mathrm{b}}$ & $1.206 \pm 0.02^{b}$ & $2.691 \pm 0.03^{\mathrm{ab}}$ & $0.677 \pm 0.03^{c}$ \\
\hline $\mathrm{NaCl}(0 \mathrm{mM})+\mathrm{CQDs} 5 \mathrm{mg} \mathrm{L}^{-1}$ & $2.033 \pm 0.08^{9}$ & $2.213 \pm 0.08^{e f}$ & $1.035 \pm 0.01^{\mathrm{ab}}$ & $0.918 \pm 0.06^{\text {de }}$ & $2.0918 \pm 0.01^{e f}$ & $0.672 \pm 0.03^{c}$ \\
\hline $\mathrm{NaCl}(0 \mathrm{mM})+\mathrm{CQDs} 10 \mathrm{mg} \mathrm{L}^{-1}$ & $2.123 \pm 0.07^{f}$ & $1.766 \pm 0.07^{f}$ & $0.976 \pm 0.08^{c}$ & $1.202 \pm 0.02^{b}$ & $2.375 \pm 0.01^{\mathrm{cd}}$ & $0.666 \pm 0.01^{d}$ \\
\hline $\mathrm{NaCl}(0 \mathrm{mM})+$ Put-CQD NPs $5 \mathrm{mg} \mathrm{L}^{-1}$ & $2.432 \pm 0.07^{c d}$ & $2.001 \pm 0.08^{\text {ef }}$ & $1.034 \pm 0.08^{\mathrm{ab}}$ & $1.215 \pm 0.02^{b}$ & $2.552 \pm 0.4^{b}$ & $0.687 \pm 0.03^{b}$ \\
\hline $\mathrm{NaCl}(0 \mathrm{mM})+$ Put-CQD NPs $10 \mathrm{mg} \mathrm{L}^{-1}$ & $2.731 \pm 0.01^{\mathrm{a}}$ & $1.564 \pm 0.04^{h}$ & $0.975 \pm 0.04^{c}$ & $1.749 \pm 0.03^{\mathrm{a}}$ & $2.748 \pm 0.03^{\mathrm{a}}$ & $0.698 \pm 0.04^{\mathrm{ab}}$ \\
\hline $\mathrm{NaCl}(100 \mathrm{mM})+$ No Treatment & $2.052 \pm 0.07^{9}$ & $3.677 \pm 0.04^{\mathrm{a}}$ & $0.861 \pm 0.04^{d}$ & $0.558 \pm 0.05^{i}$ & $1.328 \pm 0.02^{\mathrm{hi}}$ & $0.667 \pm 0.01^{d}$ \\
\hline $\mathrm{NaCl}(100 \mathrm{mM})+$ Put $5 \mathrm{mg} \mathrm{L}^{-1}$ & $2.227 \pm 0.02^{e f}$ & $3.375 \pm 0.06^{\mathrm{ab}}$ & $1.028 \pm 0.02^{b}$ & $0.659 \pm 0.01^{h}$ & $1.746 \pm 0.01^{\mathrm{g}}$ & $0.696 \pm 0.03^{\mathrm{ab}}$ \\
\hline $\mathrm{NaCl}(100 \mathrm{mM})+$ Put $10 \mathrm{mg} \mathrm{L}^{-1}$ & $2.263 \pm 0.08^{e}$ & $2.809 \pm 0.03^{b c}$ & $1.071 \pm 0.09^{\mathrm{ab}}$ & $0.805 \pm 0.02^{\mathrm{fg}}$ & $1.985 \pm 0.05^{f}$ & $0.695 \pm 0.01^{\mathrm{ab}}$ \\
\hline $\mathrm{NaCl}(100 \mathrm{mM})+\mathrm{CQDs} 5 \mathrm{mg} \mathrm{L}^{-1}$ & $2.131 \pm 0.09^{f}$ & $3.185 \pm 0.07^{\mathrm{ab}}$ & $1.138 \pm 0.07^{\mathrm{a}}$ & $0.669 \pm 0.04^{h}$ & $1.272 \pm 0.03^{i}$ & $0.692 \pm 0.04^{\mathrm{ab}}$ \\
\hline $\mathrm{NaCl}(100 \mathrm{mM})+\mathrm{CQDs} 10 \mathrm{mg} \mathrm{L}^{-1}$ & $2.205 \pm 0.05^{\mathrm{ef}}$ & $2.953 \pm 0.09^{b}$ & $0.867 \pm 0.03^{d}$ & $0.746 \pm 0.01^{\mathrm{g}}$ & $1.343 \pm 0.01^{h}$ & $0.675 \pm 0.03^{c}$ \\
\hline $\mathrm{NaCl}(100 \mathrm{mM})+$ Put-CQD NPs $5 \mathrm{mg} \mathrm{L}^{-1}$ & $2.403 \pm 0.06^{d}$ & $2.805 \pm 0.07^{\mathrm{bc}}$ & $0.986 \pm 0.01^{c}$ & $0.855 \pm 0.03^{f}$ & $2.037 \pm 0.04^{\mathrm{ef}}$ & $0.709 \pm 0.02^{\mathrm{ab}}$ \\
\hline $\mathrm{NaCl}(100 \mathrm{mM})+$ Put-CQD NPs $10 \mathrm{mg} \mathrm{L}^{-1}$ & $2.506 \pm 0.09^{b}$ & $2.738 \pm 0.05^{c}$ & $0.813 \pm 0.06^{\mathrm{e}}$ & $0.905 \pm 0.06^{\mathrm{e}}$ & $2.201 \pm 0.03^{e}$ & $0.721 \pm 0.02^{\mathrm{a}}$ \\
\hline
\end{tabular}

under both control-stress and stress conditions (Table 3).

\section{Cellular damage indicators}

Salinity caused significant enhancement $(P \leq 0.05)$ in electrolyte leakage (EL). All priming treatments significantly decreased EL values under both control and stress conditions with optimal protection being achieved following 5 $\mathrm{mg} \mathrm{L}^{-1}$ Put-CQD NPs priming treatment (Fig. 3).

As expected, MDA and $\mathrm{H}_{2} \mathrm{O}_{2}$ contents increased after imposing salinity stress, while priming treatments significantly decreased $(P \leq 0.05)$ MDA and $\mathrm{H}_{2} \mathrm{O}_{2}$ contents under both control and stress conditions. In general, $10 \mathrm{mg} \mathrm{L}^{-1}$ Put-CQD NP priming treatment provided optimal results in terms of amelioration of MDA and $\mathrm{H}_{2} \mathrm{O}_{2}$ increases under both conditions (Fig. 4a, b).

\section{Proline and total phenolic compounds}

Salinity significantly enhanced $(\mathrm{P} \leq 0.05)$ proline content and total phenolic compounds of grape (Fig. 5). Under control and stress conditions, priming treatments

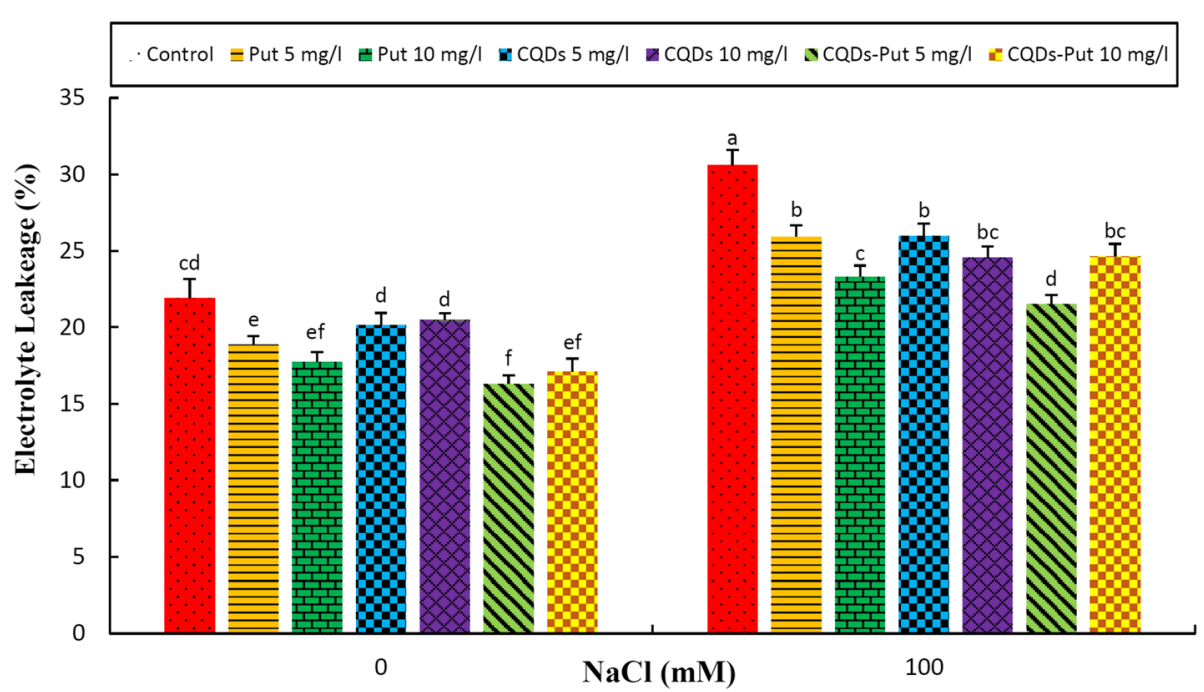

Fig. 3 Effect of different concentrations of Put, CQDs, and Put-CQD NPs in electrolyte leakage (EL) of Vitis vinifera cv. 'Sultana' under salinity stress (CQDs, carbon quantum dots; Put, putrescine, Put-CQD NPs, carbon quantum dots functionalized by putrescine nanoparticles). Different letters indicate significant differences based on Duncan's post-hoc analysis at $P \leq 0.05$ 


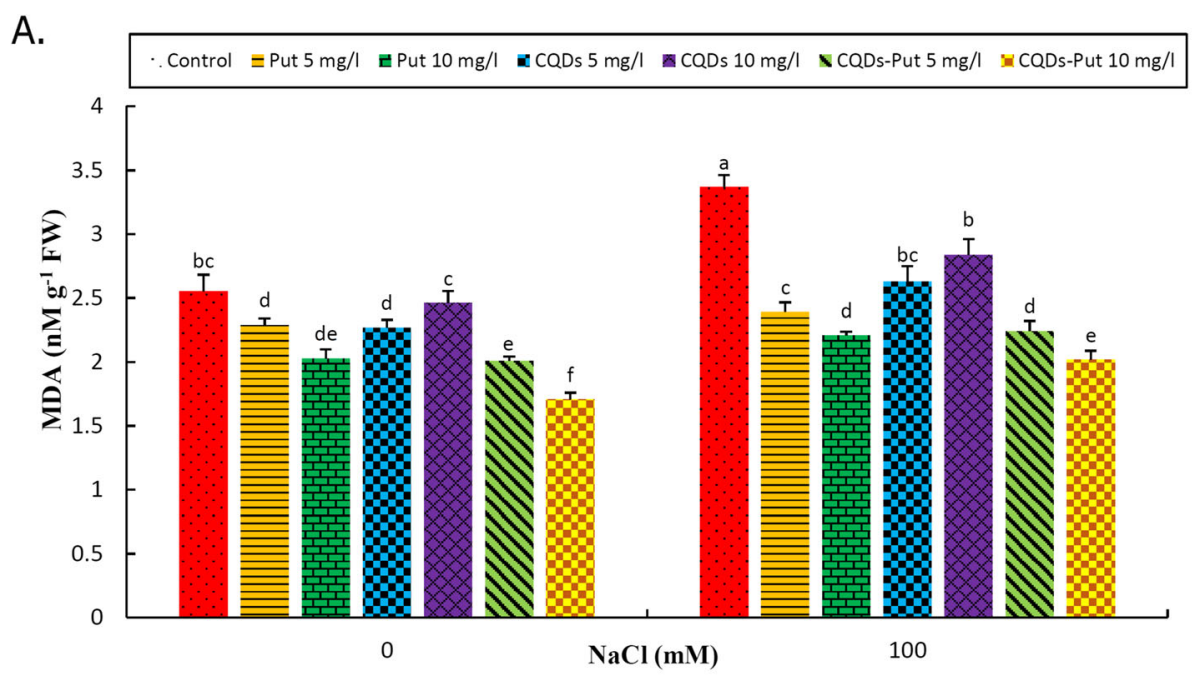

B.

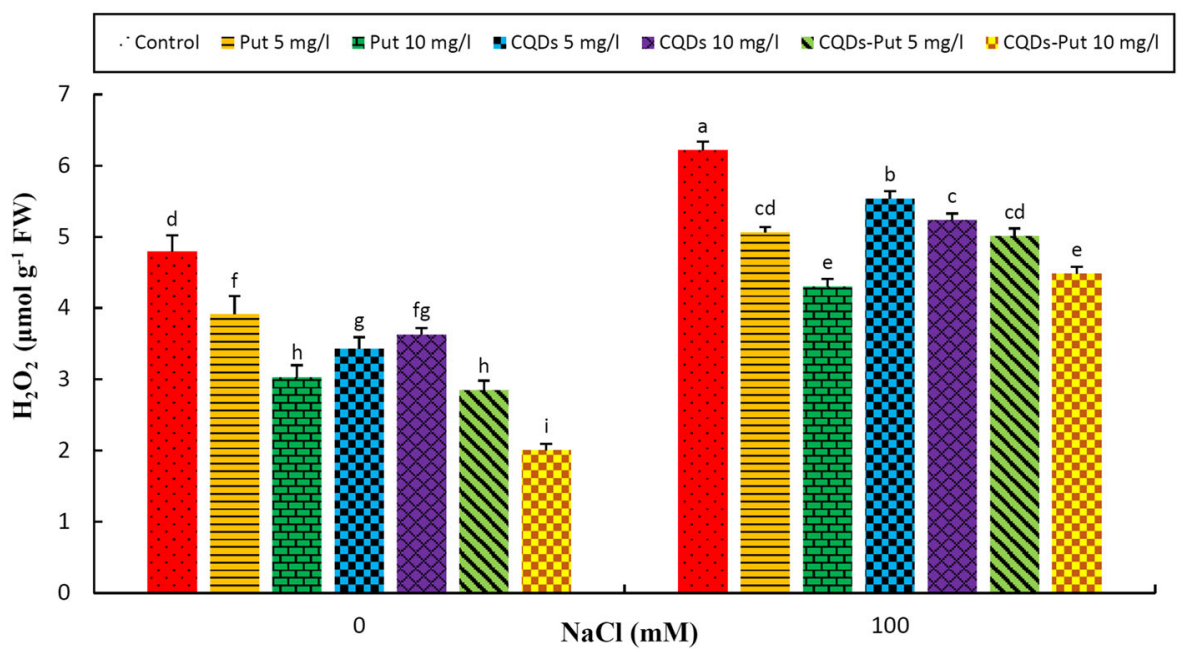

Fig. 4 Effect of different concentrations of Put, CQDs, and Put-CQD NPs in MDA (a) and $\mathrm{H}_{2} \mathrm{O}_{2}$ (b) content of Vitis vinifera cv. 'Sultana' under salinity stress (CQDs, carbon quantum dots; Put, putrescine, Put-CQD NPs, carbon quantum dots functionalized by putrescine nanoparticles). Different letters indicate significant differences based on Duncan's post-hoc analysis at $P \leq 0.05$

including Put at $10 \mathrm{mg} \mathrm{L}^{-1}$ and Put-CQD NPs at 5 and $10 \mathrm{mg} \mathrm{L}^{-1}$ concentrations significantly increased proline content, while the other treatment exerted no significant effect in this regard. The highest proline content was recorded following $10 \mathrm{mg} \mathrm{L}^{-1}$ Put-CQD NP treatment under both conditions (Fig. 5a). Under control conditions, all priming treatments (with the exception of Put and CQDs at $5 \mathrm{mg} \mathrm{L}^{-1}$ concentrations) increased total phenolics. Under salinity conditions, $10 \mathrm{mg} \mathrm{L}^{-1}$ CQDs and 5 and $10 \mathrm{mg} \mathrm{L}^{-1}$ Put-CQD NP priming treatments enhanced phenolic content; the other treatments had no effect compared with unprimed grapevine under salinity. The highest proline content was recorded following $10 \mathrm{mg} \mathrm{L}^{-1}$ PutCQD NP priming grapes under salt stress condition (Fig. $5 b)$.

\section{Antioxidant enzymatic activities}

Antioxidant enzymatic activities (CAT, APX, GP and SOD) were enhanced after imposing salinity (Fig. 6). All priming treatments increased CAT enzyme activity under control and stress conditions, with the highest activity of the enzyme being recorded following $10 \mathrm{mg} \mathrm{L}^{-1}$ Put-CQD NP application, followed by Put at $10 \mathrm{mg} \mathrm{L}^{-1}$ concentration under salinity conditions (Fig. 6a).

Considering APX enzyme, all priming treatments enhanced its activity under control conditions with optimal results following 5 and $10 \mathrm{mg} \mathrm{L}^{-1}$ Put-CQD NP treatments. Under salinity conditions, only 5 and $10 \mathrm{mg} \mathrm{L}^{-1}$ Put-CQD NP priming significantly increased APX activity, while other priming treatments had no significant 

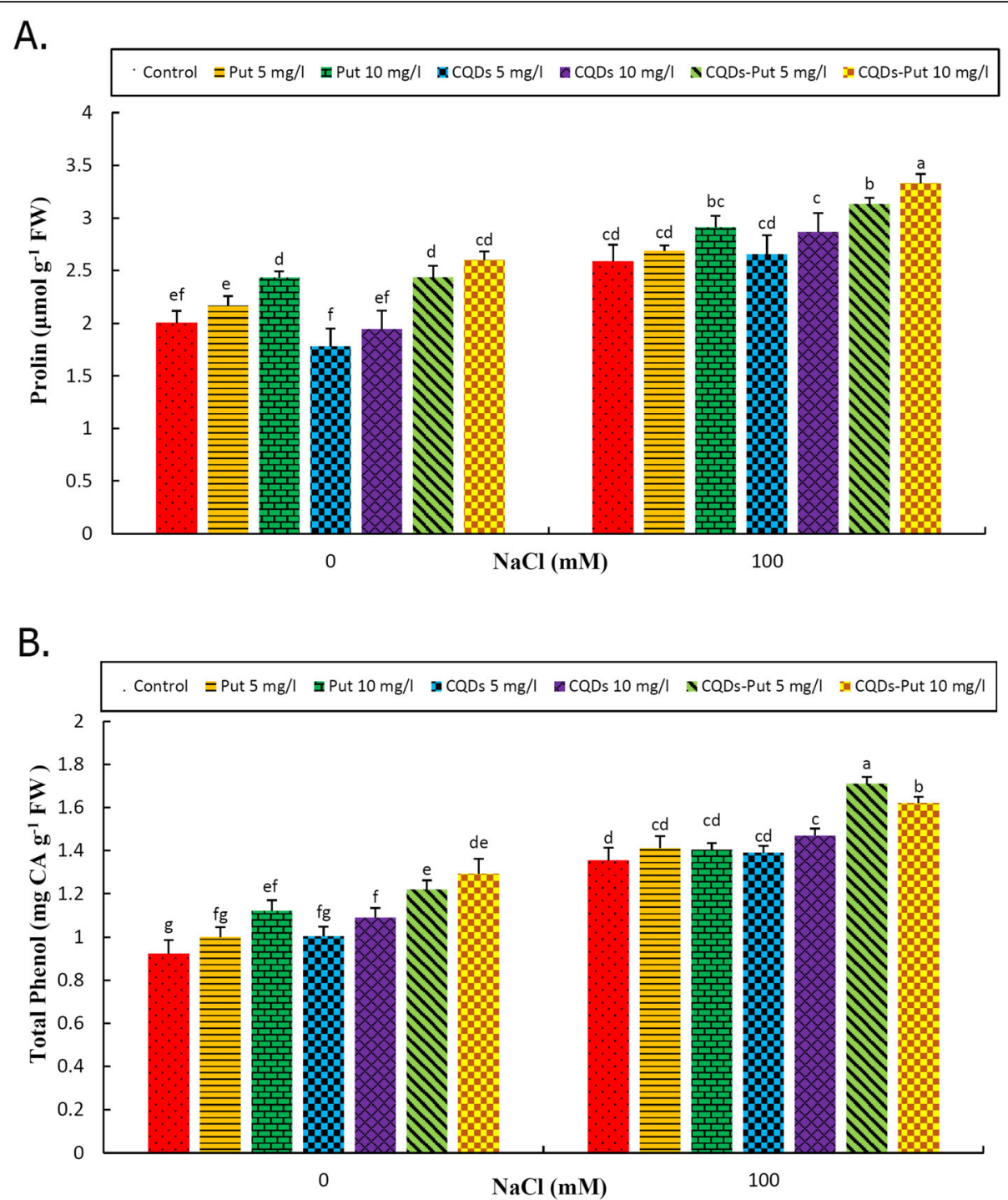

Fig. 5 Effect of different concentrations of Put, CQDs, and Put-CQD NPs in proline (a) and total phenolic content (b) of Vitis vinifera cv. 'Sultana' under salinity stress (CQDs, carbon quantum dots; Put, putrescine, Put-CQD NPs, carbon quantum dots functionalized by putrescine nanoparticles). Different letters indicate significant differences based on Duncan's post-hoc analysis at $P \leq 0.05$

effect compared with unprimed grapevines under salinity (Fig. 6b).

Under control conditions, all treatments increased GP enzyme activity with optimal activity being recorded following $10 \mathrm{mg} \mathrm{L}^{-1}$ Put-CQD NP treatment. Most treatments, enhanced GP activity under salt stress, with the exception of $5 \mathrm{mg} \mathrm{L}^{-1}$ Put and $5 \mathrm{mg} \mathrm{L}^{-1}$ CQD treatments which demonstrated similar GP activity in unprimed grapevines under salinity. The highest activity was recorded following application of $5 \mathrm{mg} \mathrm{L}^{-1}$ PutCQD NPs (Fig. 6c).

SOD enzymatic activity increased significantly $(P \leq$ $0.05)$ following application of all priming treatments under control condition. The highest activity was recorded following $10 \mathrm{mg} \mathrm{L}^{-1}$ Put-CQD NP treatment. Under salinity conditions, all treatments (except $5 \mathrm{mg} \mathrm{L}^{-1}$ Put) enhanced SOD activity, with optimal results being recorded following 5 and 10 $\mathrm{mg} \mathrm{L}^{-1}$ Put-CQD NP priming treatments (Fig. 6d).

\section{Discussion}

Salinity negatively affects plant growth and its physiological, metabolic and biological routes through induced osmotic stress [2]. Therefore, salinity decreases leaf FW and DW (e.g., [23]), in accordance with present findings. The positive effect of some NPs on agronomic traits under salinity stress has been previously reported (e.g., $[12,13])$, in line with Put-CQD NPs effects. Priming 

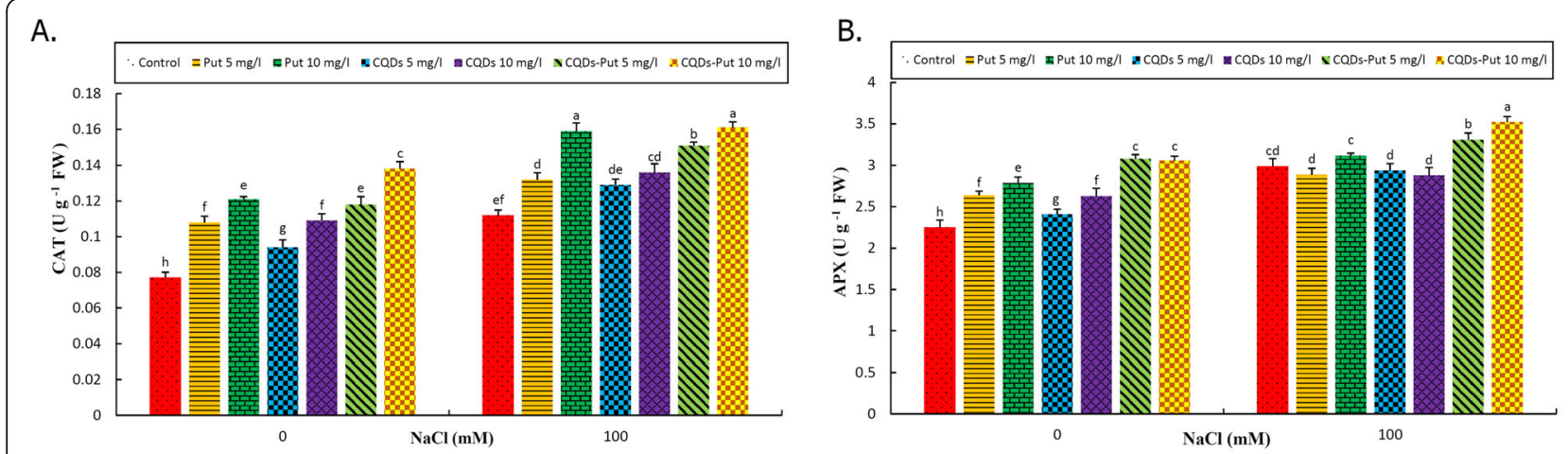

C.

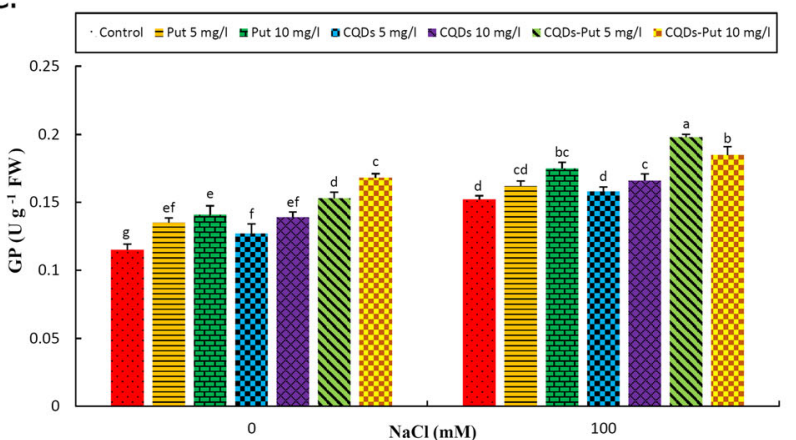

D.

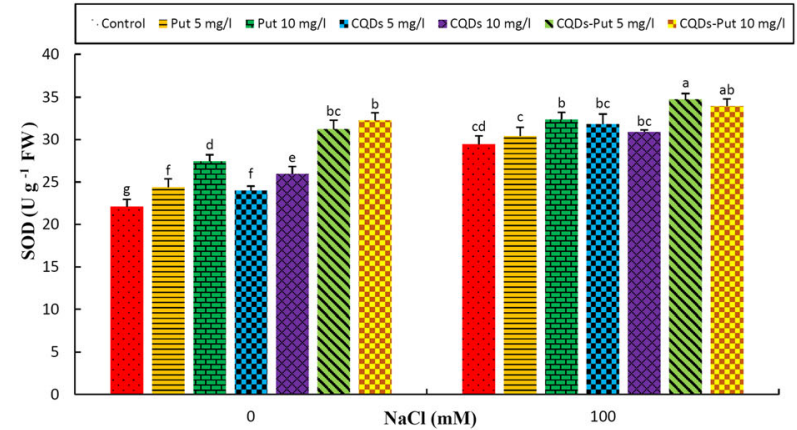

Fig. 6 Effect of different concentrations of Put, CQDs, and Put-CQD NPs in antioxidant enzymatic activities of CAT (a), APX (b), GP (c), and SOD (d) of Vitis vinifera cv. 'Sultana' under salinity stress (CQDs, carbon quantum dots; Put, putrescine, Put-CQD NPs, carbon quantum dots functionalized by putrescine nanoparticles). Different letters indicate significant differences based on Duncan's post-hoc analysis at $P \leq 0.05$

with Put also exerted positive effects regarding leaf FW and DW correlating with enhanced salt tolerance of grapevine. Similar findings, supporting the protective role of Put or other PAs in plants under salinity stress, were previously reported $([23,26,28])$. Put effects in improvement of growth parameters could be described via its polycationic nature and regulation of ion metabolism that enhance tolerance to salinity [28]. Therefore, positive effects of Put-CQD NPs could be attributed to the above-mentioned reasons.

In general, salinity enhances $\mathrm{Na}^{+}$and reduces $\mathrm{K}^{+}$ions in plants due to excessive amounts of $\mathrm{Na}^{+}$in soil or nutrient solution that imbalance ion uptake by plants [27, $28,31]$. Beneficial effect of Put or other PAs on reducing $\mathrm{Na}^{+}$and increasing $\mathrm{K}^{+}$contents in plants under salinity was previously reported [23, 26]. This impact could be described through regulation of ion channel activity in root cells by PAs (e.g. Put) to repress $\mathrm{Na}^{+}$influx into roots and enhance $\mathrm{K}^{+}$influx from roots to shoots. In addition, reduction in the activity of plasma membranebound $\mathrm{H}^{+}$-ATPase via applied salinity could be alleviated by PA application, which then decreases $\mathrm{Na}^{+}$and increases $\mathrm{K}^{+}$contents in plants grown under salinity conditions [26]. Another possible reason for positive effect of Put in this regard might be through its role in stabilizing membranes and maintaining cation-anion balance
[28]. Put acts as a signaling regulator responsible for inward rectification of $\mathrm{K}^{+}$channels [28]. Mozafari et al. [31] reported positive effect of iron-NPs on decreasing $\mathrm{Na}^{+}$and increasing $\mathrm{K}^{+}$contents in plants under salinity conditions. To our knowledge, there is no prior art showing the effect of carbon-based NPs on $\mathrm{Na}^{+}$and $\mathrm{K}^{+}$ contents in plants under salt stress conditions. This is therefore the first report demonstrating the positive effect of Put-CQD NPs on reducing $\mathrm{Na}^{+}$and enhancing $\mathrm{K}^{+}$contents in plants under salt stress conditions.

Salinity causes a drop in photosynthetic pigment content including chl $a, b$ and carotenoids. Chl content declines through decrease in chl biosynthesis and increase in its degradation/turnover. Breakdown of photosynthetic pigments could occur via the accumulation of toxic ions in chloroplasts and enhancing oxidative stress in plants after imposing salinity stress [33]. In addition, inhibition of photochemical reactions and downregulation of chloroplast-encoded genes due to salinity cause lead to a decrease in chl content [26]. Reduction in chl $a$ and $b$ content of plants under salinity stress conditions was noticed by Hatami et al. [33] and Gohari et al. [11-13], in accordance with the current study. PAs could reverse these negative effects of salinity by stabilizing oligomeric photosynthetic proteins and in particular the chl $a / b$-binding proteins displaying protease action 
during stress [26]. Although CQDs treatments enhanced chl $a, b$ and total chlorophyll, further increase in CQDs concentration subsequently lowered their values [34]. Furthermore, Gohari et al. [12] reported positive effect of modified-MWCNTs in chl $a$ and $b$ content. The current study reported positive effect of CQDs in chl $a$ and $b$ content under salinity conditions. Salinity stress is known to alter biosynthesis and accumulation of secondary metabolites like carotenoids [35]. Decrease in carotenoids of grapevine under salt stress might be due to induction of the pathway for abscisic acid production in order to modulate plant growth [32]. Increased carotenoids improve plant tolerance to stress condition due to quenching of ROS and preserving chloroplast from photo oxidation under stress conditions through their non-enzymatic antioxidant function [36]. Positive effect of some NPs such as $\mathrm{TiO}_{2}$ and MWCNT NPs in carotenoid content has also been previously noticed in plants grown under stress conditions [12, 13], thus supporting the encouraging impacts of Put-CQD NPs in carotenoid content in grapevine under salinity conditions.

Chlorophyll fluorescence parameters are solid markers for the evaluation of physiological properties of plants and the detection of stress effects. A significant decrease in chlorophyll fluorescence parameters may be caused by the dissipation of a major proportion of light energy as heat under salt stress [37], previously reported by Netondo et al. [38] and Gohari et al. [12, 13]. Some studies indicated chlorophyll fluorescence adjustment following NP application [12, 13]. Increase in chl $a$ and $b$ could occur through enhancement in light energy of PSI absorbed by chloroplast membrane to be transferred to PSII, promotion of light energy conversion to electron energy and electron transport and acceleration of water photolysis and oxygen evolution [39]. Another probable reason might be the increased absorption of carbon dioxide in plants and RuBisCO enzyme activity with an important role in photosynthesis and chlorophyll fluorescence parameters [40].

Electrolyte leakage is a reliable cellular damage indicator that could identify any damage to cell membrane integrity [41]. Salinity increases EL value by disrupting cell membrane integrity. PA application lowered EL value of plants under salinity through their polycationic nature causing direct binding to negatively charged membrane phospholipid head groups that maintain membrane function and stabilized it under stress conditions [26, 28]. Furthermore, SWCNT and MWCNT application at lower doses decreased EL value and increased cell membrane stability under salinity condition [12,33], while reduction in EL was also reported after $\mathrm{SiO}_{2} \mathrm{NP}$ application [42].

ROS generation at high concentrations has destructive impacts such as lipid peroxidation that disturbs membrane integrity and enhances MDA content [43]. Increased MDA content was reported in plants under salinity. Exogenous application of PAs decreased MDA levels in plants under salinity [24, 26, 27, 44]. Furthermore, graphene QD application at low doses decreased MDA content [45]. Mozafari et al. [31] observed similar mitigating effect of iron-NPs on decreasing MDA content of grapevine under salinity. It is likely that stabilizing membrane integrity by Put and CQDs and their conjugated form (Put-CQD NPs) could (at least in part) justify the decrease in MDA content under salinity condition.

$\mathrm{H}_{2} \mathrm{O}_{2}$, a key regulator for multiple processes linked with growth, development and stress protection [46], has binary effects depending on its concentration. At low concentration, it acts as a signaling molecule needed for initiation of resistance mechanisms to biotic and abiotic stresses, while it results in oxidative stress [47] and programmed cell death when at high concentrations. $\mathrm{H}_{2} \mathrm{O}_{2}$ leads to lipid peroxidation through hydroxyl radical formation [48]. Increase in $\mathrm{H}_{2} \mathrm{O}_{2}$ content of grapevine under salinity was previously reported [31]. The observed decrease in $\mathrm{H}_{2} \mathrm{O}_{2}$ content following Put priming of plants under salinity comes in agreement with previous reports $[24,26]$. This ameliorative effect could be described through the scavenging of free radicals and protection of proteins by PAs [28]. In fact, PAs could reverse salinity effects like ROS generation (e.g., $\mathrm{H}_{2} \mathrm{O}_{2}$ ), lipid peroxidation and corresponding MDA production [26]. Increase in proline by the treatments application could describe decrease in $\mathrm{H}_{2} \mathrm{O}_{2}$ values as increased proline could reduce $\mathrm{H}_{2} \mathrm{O}_{2}$ and other radicals either itself or by activating antioxidant enzymes activities (e.g., SOD, APX, GP and CAT) [49]. Mozafari et al. [31] reported that iron-NPs decrease $\mathrm{H}_{2} \mathrm{O}_{2}$ content in grapevine plants under salinity via increasing the antioxidant enzyme activities.

Proline is an osmolyte, metal chelating, antioxidant and signaling molecule [50]. Proline accumulates under abiotic stresses due to its role as osmotic regulator and ROS detoxifier that preserves membrane integrity, subcellular structures, antioxidant enzymatic activities and protein structure $[31,50]$. Increase in proline content in salt-stressed grapevine has been previously recorded [31], likely due to a decrease in proline oxidation and increase in its biosynthesis [51]. Exogenous PA application including Put increased proline accumulation in plants under salinity stress $[26,44]$. This enhancement could be considered as a mechanism to protect plants against salinity since proline is an osmolyte, storage material for nitrogen through stress, ROS scavenger and a modulator for $\mathrm{NADP}^{+} / \mathrm{NADPH}$ redox state. Increased proline content following PA application leads to protection of intercellular macromolecules, osmotic adoptability and 
the scavenging of hydroxyl radicals resulting in salt tolerance [26]. Iron-NPs were also shown to enhance proline content of grapevine under control and salinity conditions [31]. This enhancement was reported following QD application, as well [45], in accordance with the current study.

Phenolics protect plant cells through their potential to act as non-enzymatic and water-soluble antioxidants. This property is achieved via quenching of ROS and free radicals [52]. Phenolics prevent ROS generation and accumulation, thus inhibiting oxidative stress and reducing its undesirable effects [16]. Most phenolics are stimulated under biotic and abiotic stresses [49], such as under salinity conditions [52]. Feng et al. [45] reported increased phenolics following application of low concentration of QDs. In addition, MWCNTs-COOH treatment enhanced plant phenolics under salinity conditions [12]. Current results demonstrated positive effect of CQDs at $10 \mathrm{mg} \mathrm{L}^{-1}$ and Put-CQDs at both concentrations probably via enhanced biosynthesis, as a line of antioxidant defense against oxidative stress imposed by $\mathrm{NaCl}$. He et al. [53] demonstrated that PA application (spermidine) induced expression of genes related to enzymatic and non-enzymatic antioxidants (e.g. phenolics compound) in plants under salinity, in partial agreement with current findings.

SOD enzymatic activity eliminates superoxide radicals by dissimulating to $\mathrm{H}_{2} \mathrm{O}_{2}$, which is then detoxified by several antioxidant enzymes (e.g. CAT, POD, APX, GP) [46]. CAT, as the main enzyme for $\mathrm{H}_{2} \mathrm{O}_{2}$ quenching, remove $\sigma$ superoxide radicals as the first step of defense against ROS to reduce oxidative stress damage. APX removes $\mathrm{H}_{2} \mathrm{O}_{2}$, similar to CAT, through the glutathioneascorbate cycle [54]. GP enzyme utilizes glutathione to detoxify $\mathrm{H}_{2} \mathrm{O}_{2}$, reducing lipids and organic hydroperoxides [46]. As salinity causes oxidative stress via ROS generation and accumulation in plant cells, antioxidant enzymes (such as CAT, SOD, GP and APX) could act as a defense mechanism for ROS detoxification. Accordingly, ROS quenching via antioxidant enzymatic activities reduces stress impacts, as an essential strategy for enhanced tolerance to stress conditions [26]. Increase in major antioxidant enzymatic activities has been well recorded in plants after imposing salinity [26, 54, 55], in agreement with current findings. PAs enhance the activity of antioxidant enzymes and non-enzymatic antioxidants (e.g. anthocyanins, flavonoids [23, 26];). In addition, PAs increase plant tolerance to salinity stress by scavenging free radicals of cells and improving cell survivability [24], as well as by inducing the expression of genes encoding antioxidant enzymes. Interestingly, PAs also act as direct free radical scavengers, due to PAs binding to antioxidant enzyme molecules [28]. Put prevents membrane peroxidation and denaturing of biomolecules under salinity through two mechanisms: First, extensive protonation of PAs at physiological $\mathrm{pH}$ enables them to scavenge free radicals directly or by conjugating to cell membrane; second, PAs by increasing antioxidant enzymatic activities and thus enhancing ROS detoxification and reducing oxidative damage. In total, these mechanisms lead to plant protection against salinity stress [23]. Such results were reported following exogenous application of PAs including Put on increased antioxidant enzymatic activities (SOD and CAT), leading to decreased ROS effects and membrane injuries [28]. In terms of the effect of nanomaterials, iron-NPs enhanced APX, SOD and POD enzymatic activities of grapevine under salinity conditions [31]. Feng et al. [45] reported enhanced CAT activity at lower graphene QD concentration, likely due to enhanced oxidative stress that reduces biosynthesis of antioxidant enzymes like CAT. Gohari et al. $[12,13]$ reported positive effect of MWCN Ts-COOH and $\mathrm{TiO}_{2}$ NPs on SOD, CAT, APX and GP enzymatic activities under both control and salt stress conditions, in line with the current finding. Therefore, significant upregulation in all antioxidant enzymatic activities following Put-CQD NP treatment could ameliorate the negative impacts of salinity, demonstrating enhanced impacts of CQDs and Put in Put-CQD NPs.

\section{Conclusion}

Taking into account the established effectiveness of Put as a stress-alleviating priming agent in plants and additionally introducing nanoparticle application as an innovative approach for improved delivery and efficiency of bioactive compounds, an advanced nanostructure was formulated using CQDs. Consequently, Put-CQD NPs were successfully applied as a priming treatment towards the improvement of grapevine cv. Sultana performance under salt stress conditions. Put-CQD NPs demonstrated improved effects compared with individual treatment of Put and CQDs, particularly at a concentration of $10 \mathrm{mg} \mathrm{L}^{-1}$ through increase in a number of agronomic, physiological and biochemical parameters., highlighting a potential synergic effect of Put and CQDs in Put-CQD NPs. In conclusion, Put-CQD NPs represent an innovative approach that could be successfully applied in grapevines to improve performance under salinity conditions, while further validation is underway to determine their effectiveness in other crop species.

\section{Methods}

Experimental site, plant materials and applied treatments The experiment was conducted in the research greenhouse of the Faculty of Agriculture, University of Maragheh, Maragheh, Iran (longitude 46 $16^{\prime} \mathrm{E}$, latitude $37^{\circ} 23^{\prime} \mathrm{N}$, altitude $1485 \mathrm{~m}$ ) as factorial experiment using a completely randomized design (CRD) in three 
replications. Two-year-old cuttings of grapevine cv. Sultana were planted in $7-\mathrm{kg}$ pots containing a mixture of coco peat and medium grain perlite in a ratio of 3:1 (each pot contained a cutting). Then, they were irrigated with $1 / 2$-strength Hoagland solution until at least eight true leaves emerged. At that point, plants were treated with the chemical priming treatments four times at $12 \mathrm{~h}$ intervals. The treatments included putrescine (Put) at two concentrations (5 and $10 \mathrm{mg} \mathrm{L}^{-1}$ ), carbon quantum $\operatorname{dot}(\mathrm{CQD}) \mathrm{NPs}$ at two concentrations (5 and $10 \mathrm{mg} \mathrm{L}^{-1}$ ) and putrescine-functionalized carbon quantum dots (Put-CQD NPs) at two concentrations (5 and $10 \mathrm{mg}$ $\left.\mathrm{L}^{-1}\right)$, each treatment in three replications. Treatments were done in combination with Hoagland solution into the culture medium of pots. The last application of priming treatments was performed $48 \mathrm{~h}$ prior to imposition of salt stress. Consequently, salinity stress at two concentrations ( 0 and $100 \mathrm{mM} \mathrm{NaCl}$ ) was imposed daily through watering with Hoagland solution and continued up to a month. All biochemical and enzymatic measurements were implemented 3 days after imposition of salt stress using fully expanded leaves. Sampled leaves were instantaneously kept into liquid nitrogen for $2 \mathrm{~min}$ and afterwards preserved at $-80^{\circ} \mathrm{C}$ freezer until measurements were carried out. Other parameters including $\mathrm{Na}^{+} / \mathrm{K}^{+}$content, photosynthetic parameters and pigments were investigated a month after salinity application. Pigments were examined via the same abovementioned sampling protocol, while leaf fresh and dry weights and photosynthetic parameters were assayed using fresh leaves. Three technical replications were used for each measurement. Control plants were irrigated simply with $1 / 2$-strength Hoagland solution.

\section{Preparation of putrescine functionalized carbon quantum dots (put-CQD NPs)}

In a $25 \mathrm{~mL}$ Teflon-lined autoclave chamber containing $10 \mathrm{~mL}$ distilled water, $0.5 \mathrm{~g}$ putrescine and $2 \mathrm{~g}$ citric acid were added and heated at $200{ }^{\circ} \mathrm{C}$ for $12 \mathrm{~h}$. After cooling the reaction temperature to room temperature, the $\mathrm{pH}$ value of resulted red-brown solution was set to 7 by $\mathrm{NaOH}$ before use and characterization. For comparison, the same procedure was used to synthesize bare CQDs.

\section{Leaf fresh and dry weights}

Five leaf samples were individually weighed for fresh weight (FW) and then kept in the oven $\left(70^{\circ} \mathrm{C}, 72 \mathrm{~h}\right)$ for dry weight (DW) measurements at the harvest stage.

\section{$\mathrm{Na}^{+}$and $\mathrm{K}^{+}$assay}

Leaf samples were randomly collected from each treatment, washed and air dried and then dried in hot-air oven at $60^{\circ} \mathrm{C}$ for $18 \mathrm{~h}$. Afterwards, the samples were ground in Willey mill and the powered samples were stored for the assay. The triacid digestion extract was used for estimation of $\mathrm{Na}^{+}$and $\mathrm{K}^{+}$by flame photometry as out-lined by Ghosh [56] and expressed in mmol kg-1 . Thereby, $\mathrm{Na}^{+} / \mathrm{K}^{+}$ratio was determined.

\section{Quantification of photosynthetic pigments (chlorophyll $a$, $b$ and carotenoids)}

Fully expanded leaves $(0.2 \mathrm{~g})$ were extracted in $0.5 \mathrm{~mL}$ acetone $(3 \% \mathrm{v} / \mathrm{v})$ and then centrifuged $(10,000 \mathrm{rpm}, 10$ $\mathrm{min}$ ) and the absorption of the obtained supernatant was recorded at $645 \mathrm{~nm}(\mathrm{Chl} b), 663 \mathrm{~nm}(\mathrm{Chl} a)$ and $470 \mathrm{~nm}$ (carotenoids) by UV-Vis spectrophotometry (UV-1800 Shimadzu, Japan). Chl $a, b$ and carotenoids contents were calculated through the equations described by Sharma et al. [57].

\section{Chlorophyll fluorescence and SPAD assay}

A dual-pam-100 chlorophyll fluorometer (Heinz Walz, Effeltrich, Germany) was used to measure chlorophyll fluorescence parameters including ${ }^{\mathrm{Fv}} / \mathrm{Fo},{ }^{\mathrm{Fv}} / \mathrm{Fm}$ and $\mathrm{Y}$ (II). The measurement was done after the plants were darkadapted for $20 \mathrm{~min}$ [58].

Five randomly selected leaves of each pot were used to determine SPAD values (leaf chlorophyll concentrations) via a SPAD-meter (502 Plus Chlorophyll Meter, Japan) [59].

\section{Electrolyte leakage (EL) assay}

For EL assay, $0.5-\mathrm{cm}$ diameter discs of fully expanded leaves were cut; the discs were then washed thrice by deionized water and incubated in ambient temperate for 24h. A conductivity meter (Hanna, HI98192) was used to measure the initial electrical conductivity (EC1) of the solution. At that time, the samples were incubated in a water bath $\left(95^{\circ} \mathrm{C}, 20 \mathrm{~min}\right)$ to release all electrolytes, cooled down to $25^{\circ} \mathrm{C}$ and their final electrical conductivity (EC2) was measured. The electrolyte leakage (EL) was calculated from following equation [60].

$$
\mathrm{EL}(\%)=(\mathrm{EC} 1 / \mathrm{EC} 2) \times 100
$$

\section{Malondialdehyde (MDA) and hydrogen peroxide $\left(\mathrm{H}_{2} \mathrm{O}_{2}\right)$ assay}

After homogenizing $0.1 \mathrm{~g}$ leaf samples with $2.5 \mathrm{~mL}$ acetic acid $(10 \% \mathrm{w} / \mathrm{v})$ and centrifuging $(15,000 \mathrm{rpm}, 20 \mathrm{~min})$, the same volume of the obtained supernatant and thiobarbituric acid $(0.5 \% \mathrm{w} / \mathrm{v})$ in trichloroacetic acid (TCA) (20\%) was incubated at $96^{\circ} \mathrm{C}$ for $30 \mathrm{~min}$ in the test tube. Samples were then placed at $0{ }^{\circ} \mathrm{C}$ for $5 \mathrm{~min}$ and centrifuged $(10,000 \mathrm{rpm}, 5 \mathrm{~min})$ and the absorbance was recorded at 532 and $600 \mathrm{~nm}$ by the spectrophotometer. MDA content was calculated using the following equation: 


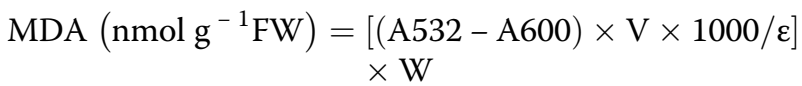

Note: $\varepsilon=$ the specific extinction coefficient $\left(155 \mathrm{mM}^{-1}\right.$ $\mathrm{cm}^{-1}$ ), $\mathrm{V}=$ the volume of crushing medium, $\mathrm{W}=$ the leaf $\mathrm{FW}, \mathrm{A} 600=$ absorbance at $600 \mathrm{~nm}$ and A $532=$ the absorbance at $532 \mathrm{~nm}$ [61].

To assay $\mathrm{H}_{2} \mathrm{O}_{2}, 0.2 \mathrm{~g}$ leaves were finely mixed with 5 $\mathrm{mL}$ trichloroacetic acid $(0.1 \% \mathrm{w} / \mathrm{v})$ in an ice bath and then centrifuged $\left(12,000 \mathrm{rpm}, 4^{\circ} \mathrm{C}, 15 \mathrm{~min}\right)$. To the obtained supernatant $(0.5 \mathrm{ml}), 0.5 \mathrm{~mL}$ potassium phosphate buffer $(\mathrm{pH} 6.8,10 \mathrm{mM})$ and $1 \mathrm{~mL}$ potassium iodide $(1 \mathrm{M})$ were added and the absorbance was recorded at $390 \mathrm{~nm}$. Finally, $\mathrm{H}_{2} \mathrm{O}_{2}$ content was calculated by standard calibration curve previously made by various $\mathrm{H}_{2} \mathrm{O}_{2}$ concentrations and expressed as $\mu \mathrm{mol} \mathrm{g}^{-1} \mathrm{FW}[62]$.

\section{Proline quantification}

To assay proline content, $0.5 \mathrm{~g}$ leaf samples were homogenized in $10 \mathrm{~mL}$ aqueous sulfosalicylic acid (3\%) in an ice bath. After centrifuging (1000 rpm, $\left.4{ }^{\circ} \mathrm{C}\right), 2 \mathrm{~mL}$ ninhydrin acid and $2 \mathrm{~mL}$ glacial acetic acid (a 1:1:1 solution) were added to $2 \mathrm{~mL}$ supernatant, finely mixed and incubated at $100^{\circ} \mathrm{C}$ for $1 \mathrm{~h}$. The reaction was stopped in an ice bath and finally $4 \mathrm{~mL}$ toluene was added and mixed vigorously $(20 \mathrm{~s})$. The mixture absorbance was recorded at $520 \mathrm{~nm}$ using the spectrophotometer. Different concentration of L-proline was used for standard curve and final calculation of proline values [63].

\section{Quantification of total phenolic compounds}

Briefly, after digesting $0.1 \mathrm{~g}$ leaf sample with $5 \mathrm{~mL} 95 \%$ ethanol, the mixture was kept in dark (24 h) and then to $1 \mathrm{~mL}$ of supernatant, $1 \mathrm{~mL} 95 \%$ ethanol and $3 \mathrm{~mL}$ distilled water were added. Next step was adding $0.5 \mathrm{~mL}$ $50 \%$ Folin-Ciocalteu solution and $1 \mathrm{~mL} 5 \%$ sodium bicarbonate, and after $1 \mathrm{~h}$ in the dark, the absorbance was recorded at $725 \mathrm{~nm}$ using the spectrophotometer. The absorbance values were converted to total phenols through standard curve made by different concentrations of gallic acid and expressed as $\mathrm{mg}$ gallic acid (GAE) $g^{-1}$ FW [64].

\section{Assay of antioxidant enzymatic activities}

Total soluble proteins and antioxidant enzymes activities were assayed through leaves formerly stored at $80^{\circ} \mathrm{C}$ freezer. All steps of enzyme extraction were carried out at $4{ }^{\circ} \mathrm{C}$ as follows: leaves $(0.5 \mathrm{~g})$ were homogenized with potassium phosphate buffer ( $\mathrm{pH} 6.8$, $100 \mathrm{mM}$ ) containing 1\% polyvinylpyrrolidone (PVP) and EDTA (4 mM) using magnetic stirrer for $10 \mathrm{~min}$. After centrifuging $(6000 \mathrm{rpm}, 20 \mathrm{~min})$, the supernatant was collected to evaluate total soluble proteins, catalase (CAT), ascorbate peroxidase (APX), superoxide dismutase (SOD) and guaiacol peroxidase (GP) enzymatic activities based on the same procedures described by Gohari et al. (2020b).

\section{Statistical analysis}

All obtained data analysis was performed by SAS software and the means of each treatment were analyzed by Duncan's multiple range test at the 95\% level of probability (SAS Institute Inc., ver. 9.1, Cary, NC, USA).

\section{Abbreviations}

CQDs: Carbon quantum dots; Put: Putrescine; Put-CQD NPS: Carbon quantum dot functionalized by putrecine nanoparticles; EL: Electrolyte leakage; $\mathrm{H}_{2} \mathrm{O}_{2}$ : Hydrogen peroxide; SOD: Superoxide dismutase; GP: Guaiacol peroxidase; APX: Ascorbate peroxidase; Chl: Chlorophyll; MDA: Malondialdehyde; CAT: Catalase; FW: Fresh weight; DW: Dry weight; MWCNTs: Multi wall carbon nanotubes; SWCNTs: Single wall carbon nanotubes

\section{Acknowledgements}

These results were from MSc thesis of Mostafa Sadeghi and we are thankful from University of Maragheh for their supports during this project.

\section{Authors' contributions}

All authors have contributed to carry out this research. GG, SP, and VF designed the experimental setup. MS performed greenhouse experiments, biochemical and physiological parameters. AA synthesized are characterized nanomaterials, EZ and SMZ performed statistical analysis. GG, SP, AA and MKB analyzed data and results, while GG, SP and VF wrote the manuscript. All authors read and approved the final manuscript.

\section{Funding}

This research did not receive any specific grant from funding agencies in the public, commercial, or not-for-profit sectors.

\section{Availability of data and materials}

The data that support the findings of this study are available from the corresponding author upon reasonable request.

\section{Declarations}

Ethics approval and consent to participate Not applicable.

\section{Consent for publication \\ Not applicable.}

\section{Competing interests}

The authors declare that they have no competing interests.

\section{Author details \\ ${ }^{1}$ Department of Horticultural Sciences, Faculty of Agriculture, University of Maragheh, Maragheh, Iran. ${ }^{2}$ Department of Horticultural Sciences, Faculty of Agriculture, University of Tabriz, Tabriz, Iran. ${ }^{3}$ Solid Tumor Research Center, Cellular and Molecular Medicine Institute, Urmia University of Medical Sciences, Urmia, Iran. ${ }^{4}$ Department of Biology, Faculty of Sciences, University of Maragheh, Maragheh, Iran. ${ }^{5}$ Department of Agricultural Sciences, Biotechnology and Food Science, Cyprus University of Technology, Limassol, Cyprus.}

Received: 24 November 2020 Accepted: 19 February 2021

Published online: 27 February 2021

\section{References}

1. Shu S, Yuan Y, Chen J, Sun J, Zhang W, Tang Y, et al. The role of putrescine in the regulation of proteins and fatty acids of thylakoid membranes under salt stress. Sci Rep. 2015;5:14390. 
2. Wani SH, Kumar V, Khare T, Guddimalli R, Parveda M, Solymosi K, et al. Engineering salinity tolerance in plants: progress and prospects. Planta. 2020;251:76.

3. Parihar $P$, Singh $\mathrm{S}$, Singh $\mathrm{R}$. Effect of salinity stress on plants and its tolerance strategies: a review. Environ Sci Pollut Res. 2015;2:4056-75.

4. Xiong L, Zhu JK. Molecular and genetic aspects of plant responses to osmotic stress. Plant Cell Environ. 2002;25(2):131-9.

5. Antoniou C, Savvides A, Christo A, Fotopoulos V. Unravelling chemical priming machinery in plants: the role of reactive oxygen-nitrogen-sulfur species in abiotic stress tolerance enhancement. Curr Opin Plant Biol. 2016; 33:101-7.

6. Savvides A, Ali S, Tester M, Fotopoulos V. Chemical priming against multiple abiotic stresses: mission possible? Trends Plant Sci. 2016;21:329-40.

7. Filippou P, Antoniou C, Fotopoulos V. The nitric oxide donor sodium nitroprusside regulates polyamine and proline metabolism in leaves of Medicago truncatula plants. Free Radic Biol Med. 2013;56:172-83.

8. Islam MM, Hoque MA, Okuma E, Banu MNA, Shimoishi Y, Nakamura Y, et al. Exogenous proline and glycinebetaine increase antioxidant enzyme activities and confer tolerance to cadmium stress in cultured tobacco cells. J Plant Physiol. 2009;166(15):1587-97.

9. Li T, Hu Y, Du X, Tang H, Shen C, Wu J. Salicylic acid alleviates the adverse effects of salt stress in Torreya grandis cv. Merrillii seedlings by activating photosynthesis and enhancing antioxidant systems. PLoS One. 2014:9(10):1-9.

10. Antoniou C, Xenofontos R, Chatzimichail G, Christou A, Kashfi K, Fotopoulos $\checkmark$. Exploring the potential of nitric oxide and hydrogen sulfide (NOSH)releasing synthetic compounds as novel priming agents against drought stress in Medicago sativa plants. Biomolecules. 2020;10:120.

11. Gohari G, Alavi Z, Esfandiari E, Panahirad S, Hajihoseinlou S. Fotopoulos, V. interaction between hydrogen peroxide and sodium nitroprusside following chemical priming of Ocimum basilicum L. against salt stress. Physiol Plant. 2020;168(2):361-73.

12. Gohari G, Mohammadi A, Akbari A, Panahirad., Dadpour MR, Fotopoulos V, et al. Titanium dioxide nanoparticles $\left(\mathrm{TiO}_{2} \mathrm{NPs}\right)$ promote growth and ameliorate salinity stress effects on essential oil profile and biochemical attributes of Dracocephalum moldavica. Sci Rep 2020;10(1):1-14.

13. Gohari G, Safai F, Panahirad S, Akbari A, Rasouli F, Dadpour MR, et al. Modified multiwall carbon nanotubes display either phytotoxic or growth promoting and stress protecting activity in Ocimum basilicum L. in a concentration-depended manner. Chemosphere. 2020;126171.

14. Khan MN, Mobin M, Abbas ZK, AlMutairi KA, Siddiqui ZH. Role of nanomaterials in plants under challenging environments. Plant Physiol Biochem. 2017:110:194-209.

15. Ioannou A, Gohari G, Papaphilippou P, Panahirad S, Akbari A, Dadpour MR, et al. Advanced nanomaterials in agriculture under a changing climate: the way to the future? Environ Exp Bot. 2020;104048.

16. Rico CM, Peralta-Videa JR, Gardea-Torresdey JL. Chemistry, biochemistry of nanoparticles, and their role in antioxidant defense system in plants. In nanotechnology and plant sciences. 2015;1-17. Springer. Cham.

17. Bai X, Purcell-Milton F, Gun'ko YK. Optical properties, synthesis, and potential applications of cu-based ternary or quaternary anisotropic quantum dots, polytypic nanocrystals, and core/shell heterostructures. Nanomaterials. 2019;9(1):85

18. Biju V, Mundayoor S, Omkumar RV, Anas A, Ishikawa M. Bioconjugated quantum dots for cancer research: present status, prospects and remaining issues. Biotechnol Adv. 2010;28(2):199-213.

19. Lim SY, Shen W, Gao Z. Carbon quantum dots and their applications. Chem Soc Rev. 2015;44(1):362-81.

20. Zhang M, Gao B, Chen J, Li YC. Effects of graphene on seed germination and seedling growth. J Nanopart Res. 2015;17(2):78.

21. Chakravarty D, Erande MB, Late DJ. Graphene quantum dots as enhanced plant growth regulators: effects on coriander and garlic plants. J Sci Food Agric. 2015;95(13):2772-8.

22. Li H, Huang J, Lu F, Liu Y, Song Y, Sun Y, et al. Impacts of carbon dots on rice plants: boosting the growth and improving the disease resistance. ACS Appl Bio Mater. 2018;1(3):663-72.

23. Zhang G, Xu S, Hu Q, Mao W, Gong Y. Putrescine plays a positive role in salt-tolerance mechanisms by reducing oxidative damage in roots of vegetable soybean. J Integr Agric. 2014;13:349-57.

24. Li S, Jin H, Zhang Q. The effect of exogenous spermidine concentration on polyamine metabolism and salt tolerance in Zoysiagrass (Zoysia japonica Steud) subjected to short-term salinity stress. Front Plant Sci. 2016;7:1221.
25. Sequera-Mutiozabal M, Antoniou C, Tiburcio AF, Alcázar R, Fotopoulos V. Polyamines: emerging hubs promoting drought and salt stress tolerance in plants. Curr Mol Biol Rep. 2017;3:28-36.

26. Saleethong P, Sanitchon J, Kong-Ngern K, Theerakulpisut P. Pretreatment with spermidine reverses inhibitory effects of salt stress in two rice (Oryza sativa L.) cultivars differing in salinity tolerance. Asian J Plant Sci. 2011;10(4): 245.

27. Quinet M, Ndayiragije A, Lefevre I, Lambillotte B, Dupont-Gillain CC, Lutts S. Putrescine differently influences the effect of salt stress on polyamine metabolism and ethylene synthesis in rice cultivars differing in salt resistance. J Exp Bot. 2010;61(10):2719-33.

28. Khoshbakht D, Asghari MR, Haghighi M. Influence of foliar application of polyamines on growth, gas-exchange characteristics, and chlorophyll fluorescence in Bakraii citrus under saline conditions. Photosynthetica. 2017; 56(2):731-42.

29. Roychoudhury A, Basu S, Sengupta DN. Amelioration of salinity stress by exogenously applied spermidine or spermine in three varieties of indica rice differing in their level of salt tolerance. J Plant Physiol. 2011;168(4):317-28.

30. Tang W, Newton RJ. Polyamines reduce salt-induced oxidative damage by increasing the activities of antioxidant enzymes and decreasing lipid peroxidation in Virginia pine. Plant Growth Regul. 2005;46(1):31-43.

31. Mozafari AA, Ghaderi N. Grape response to salinity stress and role of iron nanoparticle and potassium silicate to mitigate salt induced damage under in vitro conditions. Physiol Mol Biol Plants. 2018;24(1):25-35.

32. Suarez DL, Celis N, Anderson RG, Sandhu D. Grape rootstock response to salinity, water and combined salinity and water stresses. Agronomy. 2019; 9(6):321.

33. Hatami M. Toxicity assessment of multi-walled carbon nanotubes on Cucurbita pepo L. under well-watered and water stressed conditions. Ecotox Environ Safe. 2017;142:274-83.

34. Wang $H$, Zhang M, Song $Y$, Li H, Huang $H$, Shao M, et al. Carbon dots promote the growth and photosynthesis of mung bean sprouts. Carbon. 2018;136:94-102.

35. Borghesi E, González-Miret ML, Escudero-Gilete ML, Malorgio F, Heredia FJ, Meléndez-Martínez AJ. Effects of salinity stress on carotenoids, anthocyanins, and color of diverse tomato genotypes. J Agric Food Chem. 2011;59(21): 11676-82.

36. Doganlar ZB, Demir K, Basak H, Gul I. Effects of salt stress on pigment and total soluble protein contents of three different tomato cultivars. Afric J Agricultural Res. 2010;5(15):2056-65.

37. Yamane K, Kawasaki M, Taniguchi M, Miyake H. Correlation between chloroplast ultrastructure and chlorophyll fluorescence characteristics in the leaves of Rice (Oryza sativa L.) grown under salinity. Plant Prod Sci. 2008;11: 139-45.

38. Netondo GW, Onyango JC. Beck E. Sorghum and salinity: II. Gas exchange and chlorophyll fluorescence of sorghum under salt stress Crop Sci 2004:44: 806.

39. Mingyu S, Fashui $\mathrm{H}$, Chao L. Effects of nano-anatase $\mathrm{TiO}_{2}$ on absorption, distribution of light, and photoreduction activities of chloroplast membrane of spinach. Biol Trace Elem Res. 2007;118:120-30.

40. Yamori W, Masumoto C, Fukayama H, Makino A. Rubisco activase is a key regulator of non-steady-state photosynthesis at any leaf temperature and, to a lesser extent, of steady-state photosynthesis at high temperature. Plant J. 2012;71(6):871-80.

41. Bajii M, Kinet JM, Lutt S. The use of the electrolyte leakage method for assessing cell membrane stability as a water stress tolerance test in durum wheat. Plant Growth Regul. 2002;36(1):61-70.

42. Siddiqui MH, Al-Whaibi MH, Faisal M, Al Shali AA. Nano-silicon dioxide mitigates the adverse effect of salt stress on Cucurbita pepo L. Environ Toxicol Chem. 2014;33:2429-37.

43. Sobahan MA, Akter N, Murata Y, Munemasa S. Exogenous proline and glycinebetaine mitigate the detrimental effect of salt stress on rice plants. Science, Engin Health Stud. 2016;10(3):38-43.

44. Tanou G, Ziogas V, Belghazi M, Christou A, Filippou P, Job D, et al. Polyamines reprogram oxidative and nitrosative status and the proteome of citrus plants exposed to salinity stress. Plant Cell Environ. 2014;37:864-85.

45. Feng P, Geng B, Cheng Z, Liao X, Pan D, Huang J. Graphene quantum dotsinduced physiological and biochemical responses in mung bean and tomato seedlings. Braz J Bot. 2019;42(1):29-41.

46. Hasanuzzaman M, Bhuyan MHMB, Zulfiqar F, Raza A, Mohsin SM, Al Mahmud J, Fujita M, Fotopoulos V. Reactive oxygen species and antioxidant 
metabolism in plants under abiotic stress: revisiting the crucial role of a universal defense regulator. Antioxidants. 2020;9:681.

47. Molassiotis A, Fotopoulos V. Oxidative and nitrosative signaling in plants: two branches in the same tree? Plant Signal Behav. 2011;6:210-4.

48. Tammam AA, Fakhry EM, El-Sheekh M. Effect of salt stress on antioxidant system and the metabolism of the reactive oxygen species in Dunaliella salina and Dunaliella tertiolecta. Afr J Biotechnol. 2011;10(19):3795-808.

49. Zheng $J$, Zhao $L Y, W u C W$, Shen B, Zhu AY. Exogenous proline reduces $\mathrm{NaCl}$-induced damage by mediating ionic and osmotic adjustment and enhancing antioxidant defense in Eurya emarginata. Acta Physiol Plant. 2015;37(9):181

50. Hayat S, Hayat Q, Alyemeni MN, Wani AS, Pichtel J, Ahmad A. Role of proline under changing environments: a review. Plant Signal Behav. 2012; 7(11):1456-66.

51. Khan MN, Siddiqui MH, Mohammad F, Khan MMA, Naeem M. Salinity induced changes in growth, enzyme activities, photosynthesis, proline accumulation and yield in linseed genotypes. World J Agric Sci. 2007;3(5): 685-95.

52. Ashraf MA, Ashraf M, Ali Q. Response of two genetically diverse wheat cultivars to salt stress at different growth stages: leaf lipid peroxidation and phenolic contents. Pak J Bot. 2010;42(1):559-65.

53. He L, Ban Y, Inoue H, Matsuda N, Liu J, Moriguchi T. Enhancement of spermidine content and antioxidant capacity in transgenic pear shoots overexpressing apple spermidine synthase in response to salinity and hyperosmosis. Phytochemistry. 2008;69(11):2133-41.

54. Gharsallah C, Fakhfakh H, Grubb D, Gorsane F. Effect of salt stress on ion concentration, proline content, antioxidant enzyme activities and gene expression in tomato cultivars. AoB Plants. 2016;8.

55. Filippou P, Bouchagier P, Skotti E, Fotopoulos V. Proline and reactive oxygen/nitrogen species biosynthesis is involved in the tolerant response of the invasive plant species Ailanthus altissima to drought and salinity. Environ Exp Bot. 2014;97:1-10.

56. Ghosh K. Methods of analysis of soils, plants, waters and fertilizers. J Ind Soc Soil Sci. 1993;41(4):814-5.

57. Sharma P, Jha AB, Dubey R, Pessarkli M. Reactive oxygen species, oxidative da mage, and antioxidative defense mechanism in plants under stressful conditions. J Bot. 2012:1-26.

58. Maxwell K, Johnson GN. Chlorophyll fluorescence-a practical guide. J Exp Bot. 2000:51(345):659-68.

59. Ling Q, Huang W, Jarvis P. Use of a SPAD-502 meter to measure leaf chlorophyll concentration in Arabidopsis thaliana. Photosynth Res. 2011; 107(2):209-14.

60. Nayyar H. Accumulation of osmolytes and osmotic adjustment in waterstressed wheat (Triticum aestivum) and maize (Zea mays) as affected by calcium and its antagonists. Environ Exp Bot. 2003;50(3):253-64.

61. Stewart RRC, Bewley JD. Lipid peroxidation associated with accelerated aging of soybean axes. Plant Physiol. 1980;65:245-58.

62. Sinha S, Saxena R, Singh S. Chromium induced lipid peroxidation in the plants of Pistia stratiotes L.: role of antioxidants and antioxidant enzymes. Chemosphere. 2005;58:595-604.

63. Bates LS, Waldren RP, Teare ID. Rapid determination of free proline for water-stress studies. Plant Soil. 1973;39(1):205-7.

64. Xu C, Zhang Y, Cao L, Lu J. Phenolic compounds and antioxidant properties of different grape cultivars grown in China. Food Chem. 2010;119:1557-65.

\section{Publisher's Note}

Springer Nature remains neutral with regard to jurisdictional claims in published maps and institutional affiliations.

\section{Ready to submit your research? Choose BMC and benefit from:}

- fast, convenient online submission

- thorough peer review by experienced researchers in your field

- rapid publication on acceptance

- support for research data, including large and complex data types

- gold Open Access which fosters wider collaboration and increased citations

- maximum visibility for your research: over $100 \mathrm{M}$ website views per year

At BMC, research is always in progress.

Learn more biomedcentral.com/submissions 